\title{
Basic exchange model: Comparison of Anderson and valence bond configuration interaction approaches and an alternative exchange expression
}

\author{
W. Van den Heuvel and L. F. Chibotaru \\ Division of Quantum Chemistry and Physical Chemistry, University of Leuven, Celestijnenlaan 200F, B-3001 Leuven, Belgium
}

(Received 15 May 2007; published 21 September 2007)

\begin{abstract}
The application of the Anderson [Phys. Rev. 115, 2 (1959)] and valence bond configuration interaction (VBCI) methods on a basic exchange model is investigated to assess their ability to calculate the exchange energy under a variety of physical situations, represented by the model parameters. The model comprises two metal orbitals and a central ligand orbital. We show that the validity of the Anderson method is not restricted to cases in which $U \ll \Delta$ if the "repulsion assisted transfer" term is included in the exchange expressions. We discuss the influence of the Coulomb repulsion in the metal and ligand orbitals on the Anderson method, pointing out some deficiencies of the Anderson orbitals. In cases of strong metal-ligand covalency, the VBCI method fails due to its perturbational character. The modified Anderson exchange performs better in some of these cases, depending on the specific situation. Based on the concept of a strict separation of covalent and exchange effects, we derive an exchange expression that overcomes all the discussed difficulties of the Anderson and VBCI methods.
\end{abstract}

DOI: 10.1103/PhysRevB.76.104424

PACS number(s): 75.30.Et, 71.70.Gm, 31.25.- -v

\section{INTRODUCTION}

Models for the magnetic exchange interaction between metal centers have found wide use ever since the seminal work of Anderson. ${ }^{1}$ Interest in exchange related phenomena has not ceased and model studies of such phenomena continue to appear in the physical ${ }^{2-14}$ as well as in the chemical $^{15-20}$ literature. Numerous proposals for improvement and extension of the Anderson superexchange theory ${ }^{1,21,22}$ have been made in the last decades (for a review see, e.g., Ref. 23 and references therein). Compared to them, the valence bond configuration interaction (VBCI) approach, ${ }^{17,24}$ represented a radically different method to describe exchange interactions, which is now widely used. The general Anderson method is based on orthogonal localized molecular orbitals on the metal sites. These orbitals include the effect of the covalent chemical bond between the metal and the ligands. Some modifications of the original Anderson model, concerning the definition of the starting orbitals, have also been proposed. ${ }^{23,25}$ The VBCI approach was successfully applied by Zaanen et al. to charge-transfer insulators, ${ }^{26}$ while to the exchange problem it was applied by Zaanen and Sawatzky $^{24}$ and Geertsma. ${ }^{27}$ Unlike Anderson's method, VBCI uses atomic starting orbitals and includes chemical bonding through configuration interaction. In Ref. 24, the authors claimed that the Anderson theory would break down when the ligand-to-metal charge-transfer energy becomes lower than the metal-to-metal charge-transfer energy. ${ }^{24} \mathrm{Al}$ though plenty of studies concerning the magnetic exchange have appeared where one of these theories is used, a quantitative analysis of the latter claim and a more general investigation of the applicability of both theories seem to be lacking. It is this gap that we want to fill with this paper. The two methods are tested by applying them to a basic model system consisting of two metal orbitals and a ligand orbital, occupied by four electrons (or two holes). Then, the capability of each approximation to reproduce the singlet-triplet splitting can be assessed by comparison with each other and with the result of an exact diagonalization within this model. It will be shown that the original Anderson approach has to be modified by including the "repulsion assisted transfer" when the condition $U \ll \Delta$ is not fulfilled. This modified Anderson exchange turns out to be more suitable than VBCI in some cases. At the same time, we identify situations in which both of these methods fail. Guided by these findings, we finally propose an alternative method based on an exact incorporation of the metal-ligand covalency. With this approach, a quantitative agreement with the exact solution can be reached for a broad range of the model parameters.

\section{MODEL SYSTEM}

The three-center system consisting of two magnetic metal ions separated by a diamagnetic ligand group has served for decades as a benchmark model to investigate the exchange interaction between metal centers. In this capacity, it has been used to test several theoretical approaches to the problem of magnetic exchange (for a review, see Ref. 23 and references therein and Ref. 17). Of particular interest is the version with four (or two) electrons in three orbitals centered on the sites since it represents a basic model in which superexchange can be studied using a minimum of parameters. Going into more detail, the model system comprises two identical metal centers separated by a central ligand center. The three sites may be placed in a linear way although this is not necessary. One "atomic" real orbital is placed on each site. The orbitals on the metal sites are of course identical, while the only condition on the ligand orbital is that it must not be orthogonal by symmetry to the metal orbitals. Without loss of generality, the three orbitals are supposed to be $s$-like from now on. Further, the orbitals are made mutually orthogonal. The starting orbitals on the metal sites are denoted as $\phi_{1}$ and $\phi_{2}$, and the orbital on the ligand as $\phi_{l}$. Four electrons are distributed over these three orbitals. The electron core that bears our model system is treated as invariant and exerts its influence through the Fock operator $\hat{F}^{c}$. The situa- 


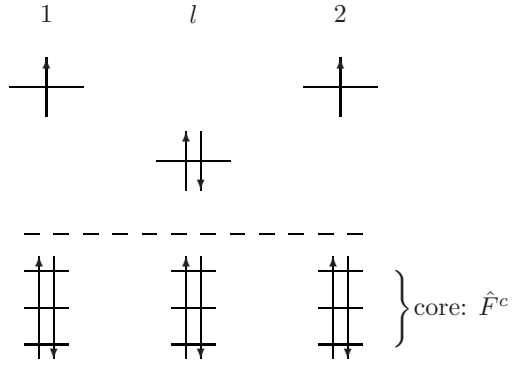

FIG. 1. The model system in the electron representation showing the configuration used in the ROHF calculation.

tion is presented schematically in Fig. 1. Interactions included are the one-electron transfer between metal and ligand $t=\left\langle\phi_{1}\left|\hat{F}^{c}\right| \phi_{l}\right\rangle=\left\langle\phi_{2}\left|\hat{F}^{c}\right| \phi_{l}\right\rangle$ and the on-site Coulomb repulsion $U=\left\langle\phi_{1}(1) \phi_{1}(2)\left|\frac{1}{r_{12}}\right| \phi_{1}(1) \phi_{1}(2)\right\rangle$ $=\left\langle\phi_{2}(1) \phi_{2}(2)\left|\frac{1}{r_{1}}\right| \phi_{2}(1) \phi_{2}(2)\right\rangle \quad$ and $U^{\prime}$ $=\left\langle\phi_{l}(1) \phi_{l}(2)\left|\frac{1}{r_{12}}\right| \phi_{l}(1) \phi_{l}(2)\right\rangle$. The electronic Hamiltonian for the system is then as follows:

$$
\begin{aligned}
H^{\mathrm{el}}= & \sum_{\sigma}\left[h_{11}\left(n_{1 \sigma}+n_{2 \sigma}\right)+h_{l l} n_{l \sigma}+\frac{1}{2} U\left(n_{1 \sigma} n_{1-\sigma}+n_{2 \sigma} n_{2-\sigma}\right)\right. \\
& \left.+\frac{1}{2} U^{\prime} n_{l \sigma} n_{l-\sigma}+t\left(a_{1 \sigma}^{\dagger} a_{l \sigma}+a_{l \sigma}^{\dagger} a_{1 \sigma}+a_{2 \sigma}^{\dagger} a_{l \sigma}+a_{l \sigma}^{\dagger} a_{2 \sigma}\right)\right],
\end{aligned}
$$

where $h_{i i}=\left\langle\phi_{i}\left|\hat{F}^{c}\right| \phi_{i}\right\rangle, n_{i \sigma}=a_{i \sigma}^{\dagger} a_{i \sigma}$, and $a_{i \sigma}^{\dagger}$ and $a_{i \sigma}$ are the electron creation and annihilation operators corresponding to the atomic orbitals. Note that there is no direct interaction between the metal sites included. To simplify further description, we pass to hole representation by interchanging creation and annihilation operators, ${ }^{28}$

$$
\begin{aligned}
H^{\text {holes }}= & 4 h_{11}+2 h_{l l}+2 U+U^{\prime}+\sum_{\sigma}\left[\left(-h_{11}-U\right)\left(n_{1 \sigma}+n_{2 \sigma}\right)\right. \\
& +\left(-h_{l l}-U^{\prime}\right) n_{l \sigma}+\frac{1}{2} U\left(n_{1 \sigma} n_{1-\sigma}+n_{2 \sigma} n_{2-\sigma}\right) \\
& \left.+\frac{1}{2} U^{\prime} n_{l \sigma} n_{l-\sigma}-t\left(a_{1 \sigma}^{\dagger} a_{l \sigma}+a_{l \sigma}^{\dagger} a_{1 \sigma}+a_{2 \sigma}^{\dagger} a_{l \sigma}+a_{l \sigma}^{\dagger} a_{2 \sigma}\right)\right],
\end{aligned}
$$

and set to zero the one-hole energy on the metals:

$$
\begin{aligned}
H^{\text {holes }}= & \sum_{\sigma}\left[\Delta n_{l \sigma}+\frac{1}{2} U\left(n_{1 \sigma} n_{1-\sigma}+n_{2 \sigma} n_{2-\sigma}\right)+\frac{1}{2} U^{\prime} n_{l \sigma} n_{l-\sigma}\right. \\
& \left.-t\left(a_{1 \sigma}^{\dagger} a_{l \sigma}+a_{l \sigma}^{\dagger} a_{1 \sigma}+a_{2 \sigma}^{\dagger} a_{l \sigma}+a_{l \sigma}^{\dagger} a_{2 \sigma}\right)\right],
\end{aligned}
$$

where $\Delta=h_{11}+U-h_{l l}-U^{\prime}$. The further discussion will be built upon the Hamiltonian in Eq. (3).

In constructing the determinantal wave functions, the central plane of symmetry is used to reduce the size of the Hamiltonian matrix and to label the wave functions according to their parity with respect to the plane $(+$ or -$)$. We have then four ${ }^{1} \Gamma_{+}$, two ${ }^{3} \Gamma_{-}$, two ${ }^{1} \Gamma_{-}$, and one ${ }^{3} \Gamma_{+}$states. The configuration with one hole on each metal center is contained in the ${ }^{1} \Gamma_{+}$and the ${ }^{3} \Gamma_{-}$sets so that the exchange interaction problem corresponds to the determination of the energy difference between the lowest ${ }^{3} \Gamma_{-}$and the lowest ${ }^{1} \Gamma_{+}$ state. Therefore, we do not need to consider the states belonging to the other two sets. In the atomic basis, the wave functions of the ${ }^{1} \Gamma_{+}$set are

$$
|\operatorname{cov} ; 00\rangle=\frac{1}{\sqrt{2}}\left(a_{1 \uparrow}^{\dagger} a_{2 \downarrow}^{\dagger}-a_{1 \downarrow}^{\dagger} a_{2 \uparrow}^{\dagger}\right)|0\rangle,
$$

$$
\begin{gathered}
|l m ; 00\rangle=\frac{1}{2}\left(a_{1 \uparrow}^{\dagger} a_{l \downarrow}^{\dagger}-a_{1 \downarrow}^{\dagger} a_{l \uparrow}^{\dagger}+a_{2 \uparrow}^{\dagger} a_{l \downarrow}^{\dagger}-a_{2 \downarrow}^{\dagger} a_{l \uparrow}^{\dagger}\right)|0\rangle, \\
|m m ; 00\rangle=\frac{1}{\sqrt{2}}\left(a_{1 \uparrow}^{\dagger} a_{1 \downarrow}^{\dagger}+a_{2 \uparrow}^{\dagger} a_{2 \downarrow}^{\dagger}\right)|0\rangle, \\
|l l ; 00\rangle=a_{l \uparrow}^{\dagger} a_{l \downarrow}^{\dagger}|0\rangle,
\end{gathered}
$$

and those of the ${ }^{3} \Gamma_{\text {_ }}$ set are (with $M_{s}=1$ )

$$
\begin{gathered}
|\operatorname{cov} ; 11\rangle=a_{1 \uparrow}^{\dagger} a_{2 \uparrow}^{\dagger}|0\rangle, \\
|l m ; 11\rangle=\frac{1}{\sqrt{2}}\left(a_{1 \uparrow}^{\dagger} a_{l \uparrow}^{\dagger}-a_{2 \uparrow}^{\dagger} a_{l \uparrow}^{\dagger}\right)|0\rangle,
\end{gathered}
$$

where the entries in the kets contain an abbreviated identification of the state, together with its $S$ and $M_{S}$ quantum numbers. The corresponding Hamiltonian matrices are ${ }^{29}$

$$
\begin{gathered}
H^{1 \Gamma_{+}}=\left(\begin{array}{cccc}
|\operatorname{cov} ; 00\rangle & |l m ; 00\rangle & |m m ; 00\rangle & |l l ; 00\rangle \\
0 & -\sqrt{2} t & 0 & 0 \\
\Delta & -\sqrt{2} t & -2 t \\
& & 0 \\
& & 2 \Delta+U^{\prime}
\end{array}\right) \\
H^{3 \Gamma_{-}}=\left(\begin{array}{cc}
|\operatorname{cov} ; 11\rangle & |\operatorname{lm} ; 11\rangle \\
0 & -\sqrt{2} t \\
\Delta
\end{array}\right)
\end{gathered}
$$

Within this model, exact exchange splittings are obtained by diagonalizing the matrices (10) and (11). One notices immediately that there is no ferromagnetism possible in this system since the ${ }^{1} \Gamma_{+}$ground state always has a larger hybridization space at its disposal than the ${ }^{3} \Gamma_{-}$ground state. It is indeed a familiar experimental fact that antiferromagnetic interactions, if present, usually dominate. ${ }^{1,21}$ Following these defining preliminaries, we can now investigate the different approximation schemes.

\section{ANDERSON AND VALENCE BOND CONFIGURATION INTERACTION TREATMENTS OF SUPEREXCHANGE}

\section{A. Anderson approach}

The Anderson method is an orbital based one. That is, a set of magnetic orbitals, localized at the metal sites, defines 
the ground and excited state configurations. Energy corrections are obtained by a perturbation approach in which excited configurations are mixed with the ground configurations. In constructing the starting orbitals, we follow closely the original Anderson recipe: ${ }^{22}$ restricted open-shell HartreeFock (ROHF) orbitals are constructed for the high-spin configuration with one hole on each metal site. This corresponds to the first (covalent) ${ }^{3} \Gamma_{-}$state in our case. Then, the singly occupied orbitals (Bloch waves in the crystal) are transformed into equivalent localized Wannier functions (the Anderson magnetic orbitals). The restricted Hartree-Fock procedure ensures the pure spin character of the determinantal states, which is of course a necessary condition to speak about the "singlet-triplet" splitting. The idea behind the orbital part of this method is that the covalent interaction between the metal and ligand orbitals is already included from the beginning, while the on-site electron repulsion remains strong enough to keep the electrons mainly localized on the metals. ${ }^{1}$ The ground configurations are those with one hole in each magnetic orbital where every hole may be spin up or spin down. The degeneracy of these configurations is guaranteed by including in the zero-order Hamiltonian only those parts of the total Hamiltonian that do not transfer holes between orbitals. These are the diagonal hole energies and the classic Coulomb repulsions. Differentiation is then brought into the ground manifold through the action of an effective perturbation Hamiltonian. This Hamiltonian reduces in every order of perturbation to the well-known effective spin Hamiltonian. For the case of only two metal centers, it takes the form $H^{\text {eff }}=2 J_{\text {exch }} \hat{\mathbf{S}}_{1} \hat{\mathbf{S}}_{2}$. The energy difference between the lowest triplet and singlet states is thus $2 J_{\text {exch }}$.

We now proceed to calculate the Anderson orbitals for our molecular system. These result from a ROHF calculation on the electron configuration with two spin-up electrons (see Fig. 1). It can be shown that exactly the same orbitals are obtained from a HF calculation on the equivalent hole configuration (see Appendix A). From the three starting orbitals, symmetry adapted orbitals are constructed:

$$
\begin{gathered}
\phi_{+}=\frac{1}{\sqrt{2}}\left(\phi_{1}+\phi_{2}\right), \\
\phi_{l}=\phi_{l}, \\
\phi_{-}=\frac{1}{\sqrt{2}}\left(\phi_{1}-\phi_{2}\right) .
\end{gathered}
$$

Minimizing the energy of the determinant with one spin-up hole on each metal [using the Hamiltonian in Eq. (3)], we arrive at the HF matrix that mixes orbitals (12) and (13):

$$
F_{+}^{\text {holes }}=\left(\begin{array}{cc}
\phi_{+} & \phi_{l} \\
0 & -\sqrt{2} t \\
& \Delta
\end{array}\right) .
$$

A few remarks should be made about this HF matrix. It depends only on two parameters $(\Delta$ and $t)$ instead of the four independent parameters that describe the system, and the eigenfunctions will only depend on the ratio of $t$ and $\Delta$. This indicates that the HF orbitals have a limited flexibility to adjust themselves to the physical situation. For instance, the HF orbitals are unaffected by an increase of the on-site Coulomb repulsion (the other parameters being the same). The repulsion parameters $U$ and $U^{\prime}$ are absent in the HF matrix because repulsion takes place only when two paired holes reside on the same atomic center. This favors our present purpose since no further iterations are necessary to solve the HF equations. Exact solutions are obtained by diagonalizing Eq. (15). The HF orbitals and their energies are

$$
\begin{gathered}
\Psi_{1+}=c_{1} \phi_{+}+c_{2} \phi_{l}, \quad \epsilon_{1+}=\frac{\Delta}{2}+\sqrt{\left(\frac{\Delta}{2}\right)^{2}+2 t^{2}}=-\sqrt{2} t \frac{c_{2}}{c_{1}}, \\
\Psi_{-}=\phi_{-}, \quad \epsilon_{-}=0, \\
\Psi_{2+}=c_{2} \phi_{+}-c_{1} \phi_{l}, \quad \epsilon_{2+}=\frac{\Delta}{2}-\sqrt{\left(\frac{\Delta}{2}\right)^{2}+2 t^{2}}=\sqrt{2} t \frac{c_{1}}{c_{2}},
\end{gathered}
$$

where (for $\Delta \geqslant 0)$

$$
\begin{gathered}
c_{1}=-\frac{1}{\sqrt{2}} \frac{t}{|t|} \sqrt{1-\frac{1}{\sqrt{1+8\left(\frac{t}{\Delta}\right)^{2}}}}, \\
c_{2}=\frac{1}{\sqrt{2}} \sqrt{1+\frac{1}{\sqrt{1+8\left(\frac{t}{\Delta}\right)^{2}}}} .
\end{gathered}
$$

It follows that $\left|c_{2}\right| \geqslant\left|c_{1}\right|$ for $\Delta \geqslant 0$ and that the orbitals depend solely on the ratio of $t$ and $\Delta$. Note also that $t \frac{c_{1}}{c_{2}}$ is always negative, regardless of the sign of $t$. Orbital $\Psi_{1+}$ [Eq. (16)] is the bonding "ligand" orbital, which is doubly occupied in the electron configuration. In the hole picture, however, it has the highest energy and is not occupied. In the configuration for which the orbitals are optimized, one spin-up hole resides in $\Psi_{-}$and one in $\Psi_{2+}$. The Anderson magnetic orbitals are then obtained by combining $\Psi_{-}$and $\Psi_{2+}$ in a symmetric and an antisymmetric way, which provides the maximal possible localization of the holes within this approach:

$$
\begin{aligned}
& A_{1}=\frac{c_{2}+1}{2} \phi_{1}-\frac{c_{1}}{\sqrt{2}} \phi_{l}+\frac{c_{2}-1}{2} \phi_{2}, \\
& A_{2}=\frac{c_{2}-1}{2} \phi_{1}-\frac{c_{1}}{\sqrt{2}} \phi_{l}+\frac{c_{2}+1}{2} \phi_{2} .
\end{aligned}
$$

The orbitals (16), (21), and (22) replace $\phi_{l}, \phi_{1}$, and $\phi_{2}$, respectively, and define the configurations in the Anderson basis. The ground configurations are $b_{1 \uparrow}^{\dagger} b_{2 \downarrow}^{\dagger}|0\rangle, b_{1 \downarrow}^{\dagger} b_{2 \uparrow}^{\dagger}|0\rangle$, $b_{1 \downarrow}^{\dagger} b_{2 \downarrow}^{\dagger}|0\rangle$, and $b_{1 \uparrow}^{\dagger} b_{2 \uparrow}^{\dagger}|0\rangle$, where the $b$ operators refer to the Anderson basis. The second quantized Hamiltonian is found by transforming Hamiltonian (3) to the new orbital basis (Appendix B). 
With these configurations as a starting point, the next step is to calculate the perturbational expression for the exchange splitting. If we have a general Hamiltonian $H=H^{0}+H^{\prime}$, where the ground state of $H^{0}$ is generally degenerate and $H^{\prime}$ denotes a perturbation, the effective perturbation Hamiltonian working in the space of the ground states of $H^{0}$ can be written as $H^{\text {eff }}=H^{(0)}+H^{(1)}+H^{(2)}+H^{(3)}+\ldots$, where the superscripts indicate the order of the perturbation after $H^{\prime}$. We then have

$$
\begin{gathered}
H^{(0)}=P_{0} H^{0} P_{0}, \\
H^{(1)}=P_{0} H^{\prime} P_{0}, \\
H^{(2)}=P_{0} H^{\prime} \frac{P}{a} H^{\prime} P_{0}, \\
H^{(3)}=P_{0} H^{\prime} \frac{P}{a} H^{\prime} \frac{P}{a} H^{\prime} P_{0}-P_{0} H^{\prime} P_{0} H^{\prime} \frac{P}{a^{2}} H^{\prime} P_{0}, \\
H^{(4)}=P_{0} H^{\prime} \frac{P}{a} H^{\prime} \frac{P}{a} H^{\prime} \frac{P}{a} H^{\prime} P_{0}-P_{0} H^{\prime} \frac{P}{a} H^{\prime} \frac{P}{a^{2}} H^{\prime} P_{0} H^{\prime} P_{0} \\
-P_{0} H^{\prime} \frac{P}{a^{2}} H^{\prime} \frac{P}{a} H^{\prime} P_{0} H^{\prime} P_{0}-P_{0} H^{\prime} \frac{P}{a} H^{\prime} P_{0} H^{\prime} \frac{P}{a^{2}} H^{\prime} P_{0} \\
+P_{0} H^{\prime} \frac{P}{a^{3}} H^{\prime} P_{0} H^{\prime} P_{0} H^{\prime} P_{0},
\end{gathered}
$$

where $P_{0}$ is the projection operator on the space of the ground states and $\frac{P}{a^{n}}=\frac{P}{\left(E_{G}^{0}-H^{0}\right)^{n}}$ with $P$ the projection operator on the space of excited states: $P=1-P_{0}$ and $E_{G}^{0}$ the unperturbed energy of the ground states. The total Hamiltonian (B4) is divided into two parts: The unperturbed Hamiltonian $\left(H^{0}\right)$ is taken as the first line of Eq. (B4) which is, as mentioned before, the part that does not transfer holes between orbitals. The rest of Eq. (B4) is considered as perturbation. Details of the procedure to evaluate the expressions in Eq. (23) are provided in Appendix C. Working out the expressions to first and second orders using Eq. (23), we get, ignoring constant terms [the new matrix elements are defined in Eq. (B3)],

$$
\begin{gathered}
H_{\text {Anderson }}^{(1)}=-2 \widetilde{J}_{12} \hat{\mathbf{S}}_{1} \hat{\mathbf{S}}_{2}, \\
H_{\text {Anderson }}^{(2)}=2\left[2 \frac{\left(\widetilde{t}_{12}+\widetilde{V}_{1222}\right)^{2}}{\widetilde{U}-\widetilde{K}_{12}}+4 \frac{\widetilde{V}_{122 l}\left(\widetilde{t}_{1 l}+\widetilde{V}_{12 l 2}\right)}{\widetilde{\Delta}+\widetilde{K}_{1 l}-\widetilde{K}_{12}}\right. \\
\left.+\frac{\left(\widetilde{V}_{1 l l 2}\right)^{2}}{2 \widetilde{\Delta}+\widetilde{U}^{\prime}-\widetilde{K}_{12}}\right] \hat{\mathbf{S}}_{1} \hat{\mathbf{S}}_{2} .
\end{gathered}
$$

Note that the indices $1,2, l$ now refer to the orbitals of the Anderson basis, $A_{1}, A_{2}$, and $\Psi_{1+}$, respectively. $H^{(1)}$ is just the ferromagnetic potential exchange between the overlapping Anderson magnetic orbitals. ${ }^{1}$ The three terms in $H^{(2)}$ result from three second-order processes: The first is the wellknown one-hole transfer between the magnetic orbitals. ${ }^{1}$ The second is a hole transfer between metal and ligand and the

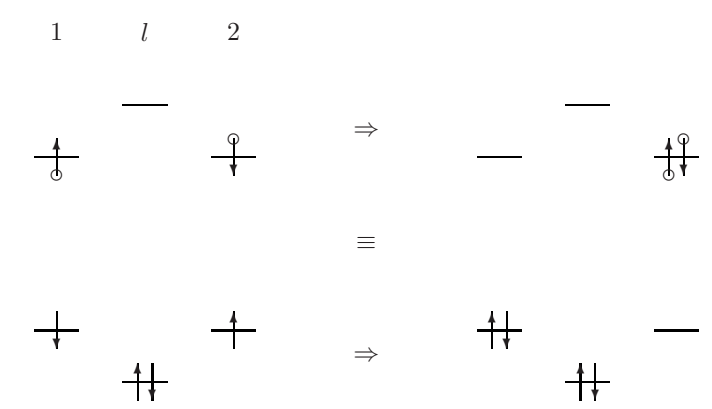

FIG. 2. Schematic representation of the antiferromagnetic onehole transfer process (upper part) and its equivalent electron transfer process (lower part).

third is a two-hole transfer between the metals and the ligand (the Nesbet term). The first term in Eq. (25) contains the kinetic exchange contribution, which was held responsible for antiferromagnetism in the original paper: ${ }^{1} \frac{4 b_{12}^{2}}{U} \hat{\mathbf{S}}_{1} \hat{\mathbf{S}}_{2}$, using the notation from that paper, where $b_{12}$ stands for the kinetic energy matrix element between the magnetic orbitals. "Kinetic energy" here means the energy of an electron in the field of the diamagnetic lattice. Expressed in terms of the present model (see Appendix B),

$$
b_{12}=\widetilde{F}_{12}^{c}+2 \widetilde{V}_{112 l}-\widetilde{V}_{1 l l 2}=-\left(\tilde{t}_{12}+2 \widetilde{V}_{1222}\right) \text {. }
$$

In the present model, $b_{12}$ is equivalent to the off-diagonal matrix element of the Fock operator for the ferromagnetic configuration (Fig. 1). We note, however, that this equivalence takes place only for this simple model. In a general case, $b_{12}$ is always understood as an off-diagonal matrix element of the Fock operator for the corresponding ferromagnetic configuration. We can see that $b_{12}$ must not be confused with $\tilde{t}_{12}$ here. The latter is the matrix element of the "onehole kinetic energy." The entanglement of terms originates from the transition from electrons to holes, where only the total matrix elements between determinantal configurations are left unchanged, apart from a possible change of sign. Consider, for example, the transfer of one electron or hole between the magnetic orbitals. This antiferromagnetic process contributes the first term to $H^{(2)}$, Eq. (25), and is shown schematically in Fig. 2. The corresponding matrix element is

$$
\left\langle A_{1} \bar{A}_{2}|H| A_{2} \bar{A}_{2}\right\rangle=\widetilde{t}_{12}+\widetilde{V}_{1222}=-\left(b_{12}+\widetilde{V}_{1222}\right),
$$

where the notation in the bracket denotes the determinantal hole configuration. Here, we see that $b_{12}$ is not the only term that transfers an electron between metal sites; $\widetilde{V}_{1222}$ represents a process which may be called repulsion assisted transfer since the transfer of one electron (hole) is mediated by the repulsion with the other electron (hole). Depending on the relative sign of $b_{12}$ and $\widetilde{V}_{1222}$, the latter can either reinforce or weaken the antiferromagnetic interaction.

\section{B. Valence bond configuration interaction}

Working out the perturbation Hamiltonians in Eq. (23) for the atomic basis system and using Eq. (3) and $H^{\prime}=-t\left(a_{1 \sigma}^{\dagger} a_{l \sigma}+a_{l \sigma}^{\dagger} a_{1 \sigma}+a_{2 \sigma}^{\dagger} a_{l \sigma}+a_{l \sigma}^{\dagger} a_{2 \sigma}\right)$, we get, up to fourth 
order (up to second or third order, there is no energy differentiation between singlet and triplet),

$$
H_{\mathrm{VBCI}}^{\mathrm{eff}}=4\left(\frac{t^{2}}{\Delta}\right)^{2}\left(\frac{1}{U}+\frac{2}{2 \Delta+U^{\prime}}\right) \hat{\mathbf{S}}_{1} \hat{\mathbf{S}}_{2} .
$$

Equation (27) is the original exchange expression found in Zaanen and Sawatzky, ${ }^{24}$ except for the ligand Coulomb repulsion $U^{\prime}$, which they did not take into account. The perturbational character of Eq. (27) requires that both $\left|\frac{t}{\Delta}\right|$ and $\left|\frac{t}{U}\right|$ are small enough to make the expansion converge and to ensure that the cutoff at fourth order is a valid approximation. The authors pointed out that whenever the transition metal compound is a charge-transfer insulator $(\Delta<U)$, Eq. (27) is to be preferred to the Anderson expression. ${ }^{24}$ Especially in the hypothetical case $U \rightarrow \infty$, Eq. (27) shows that there is still an antiferromagnetic interaction.

\section{Comparison}

We are now in a position to compare the different approximation schemes in a quantitative way. For this purpose, the triplet-singlet exchange splitting is plotted as a function of the parameter $t$, which represents the metal-ligand hybridization interaction in the atomic basis. The exact exchange splitting is calculated by exact diagonalization of the matrices (10) and (11). With regard to the Anderson approach, the original method only applied to the half filled magnetic orbitals on the metals, i.e., it did not consider the filled ligand orbital in the perturbation expressions. Still several ways of approximation are possible here. There is the purely kinetic part which ignores the first-order potential exchange $\left(H_{\text {Anderson }}^{\mathrm{A}}\right)$, the potential plus the kinetic part $\left(H_{\text {Anderson }}^{\mathrm{B}}\right)$, and the potential plus the kinetic plus the repulsion assisted part $\left(H_{\text {Anderson }}^{\mathrm{C}}\right)$ :

$$
\begin{gathered}
H_{\text {Anderson }}^{\mathrm{A}}=\frac{4 b_{12}^{2}}{\widetilde{U}-\tilde{K}_{12}} \hat{\mathbf{S}}_{1} \hat{\mathbf{S}}_{2}, \\
H_{\text {Anderson }}^{\mathrm{B}}=\left[-2 \widetilde{J}_{12}+\frac{4 b_{12}^{2}}{\widetilde{U}-\widetilde{K}_{12}}\right] \hat{\mathbf{S}}_{1} \hat{\mathbf{S}}_{2}, \\
H_{\text {Anderson }}^{\mathrm{C}}=\left[-2 \widetilde{J}_{12}+\frac{4\left(b_{12}+\widetilde{V}_{1222}\right)^{2}}{\widetilde{U}-\widetilde{K}_{12}}\right] \hat{\mathbf{S}}_{1} \hat{\mathbf{S}}_{2} .
\end{gathered}
$$

Higher forms, including the effect of the ligand orbital, are the perturbation up to complete second order, $H^{(1)}+H^{(2)}$ [Eqs. (24) and (25)], and likewise up to third, fourth, etc., orders. Equation (30) was also obtained by Hay et al. ${ }^{31}$ using direct configuration interaction within $A_{1}$ and $A_{2}$ but they considered the repulsion assisted transfer term $\widetilde{V}_{1222}$ unimportant, reducing the exchange expression to Eq. (29).

We are not concerned here with the application of the discussed theoretical approaches to real experimental cases, but merely want to compare them quantitatively as applied to one and the same model system. The parameters take values in arbitrary energy units.

\section{Cases for which $\left|\frac{t}{\Delta}\right| \ll 1,\left|\frac{t}{U}\right| \ll 1$}

In this region of parameters, it is clear that the VBCI approximation of the exchange splitting [Eq. (27)] is valid. On the other hand, it is not at once apparent how the Anderson-based expressions relate to the VBCI equation. An expansion of the elements of the Anderson equations, collecting terms of $\left(\frac{t^{2}}{\Delta}\right)^{2}$ and neglecting higher powers, will clarify this situation. ( $U$ and $U^{\prime}$ are supposed to be of the same order of magnitude as $\Delta$.) We get

$$
\frac{4 b_{12}^{2}}{\widetilde{U}-\widetilde{K}_{12}} \approx 4\left(\frac{t^{2}}{\Delta}\right)^{2} \frac{\left(1+\frac{U}{\Delta}\right)^{2}}{U}=4\left(\frac{t^{2}}{\Delta}\right)^{2}\left(\frac{1}{U}+\frac{2}{\Delta}+\frac{U}{\Delta^{2}}\right),
$$

$$
\begin{gathered}
-2 \widetilde{J}_{12}+\frac{4 b_{12}^{2}}{\widetilde{U}-\widetilde{K}_{12}} \approx 4\left(\frac{t^{2}}{\Delta}\right)^{2}\left[\frac{1}{U}+\frac{1}{\Delta}\left(1-\frac{U^{\prime}}{2 \Delta}\right)+\frac{1}{\Delta}+\frac{3 U}{4 \Delta^{2}}\right] \\
-2 \widetilde{J}_{12}+\frac{4\left(b_{12}+\widetilde{V}_{1222}\right)^{2}}{\widetilde{U}-\widetilde{K}_{12}} \approx 4\left(\frac{t^{2}}{\Delta}\right)^{2}\left[\frac{1}{U}+\frac{1}{\Delta}\left(1-\frac{U^{\prime}}{2 \Delta}\right)\right]
\end{gathered}
$$

The last term in Eq. (33) is the first part of a Taylor expansion of $\frac{1}{\Delta\left(1+\frac{U^{\prime}}{2 \Delta}\right)}=\frac{2}{2 \Delta+U^{\prime}}$. Therefore, $H_{\text {Anderson }}^{\mathrm{C}}$ is equivalent to the VBCI expression [Eq. (27)] for $\frac{U^{\prime}}{2 \Delta} \ll 1$. Now, we add the other second-order terms of Eq. (25). The second one is of the order $\left(\frac{t}{\Delta}\right)^{6}$ and can be neglected. Addition of the third term of Eq. (25) to Eq. (33) yields exactly

$$
H_{\text {Anderson }}^{(1)}+H_{\text {Anderson }}^{(2)} \approx 4\left(\frac{t^{2}}{\Delta}\right)^{2}\left(\frac{1}{U}+\frac{2}{2 \Delta+U^{\prime}}\right) \hat{\mathbf{S}}_{1} \hat{\mathbf{S}}_{2},
$$

which is just the VBCI expression [Eq. (27)]. This shows that - for a relative small $t$ - a second-order perturbation calculation based on the Anderson orbitals, including the metalto-metal transfer and the double ligand-to-metal excitation, is equivalent to an atomic orbital based calculation including the same processes in fourth order of perturbation. By using a different approach, Geertsma has obtained a similar result. ${ }^{23}$ The approximate expressions [Eqs. (31) and (32)] show that the "conventional" Anderson approach [that is, including only kinetic or kinetic and potential exchange $\left(H_{\text {Anderson }}^{\mathrm{A}}\right.$ and $\left.\left.H_{\text {Anderson }}^{\mathrm{B}}\right)\right]$ is only correct when $U, U^{\prime} \ll \Delta$, which is not the case in most materials. We note that the term $\propto \frac{U}{\Delta^{2}}$, which cannot be considered small, comes in Eq. (31) via the transfer amplitude $b_{12}$ [Eq. (B8) and Table I]:

$$
b_{12} \approx \frac{t^{2}}{\Delta}\left(1+\frac{U}{\Delta}\right) .
$$

Zaanen and Sawatzky ${ }^{24}$ discussed Eq. (27) as being composed of an Anderson term $\left(4 \frac{\left(t^{2} / \Delta\right)^{2}}{U}\right)$ and an additional term. They approximated $b_{12} \approx \frac{t^{2}}{\Delta}$. However, using the correct ap- 
TABLE I. Exact expressions of the matrix elements of the hole Hamiltonian (B4) in the Anderson basis in terms of the orbital coefficients $c_{1}$ and $c_{2}$ and the model parameters $t, \Delta, U$, and $U^{\prime}$ and approximate expressions for $\left|\frac{t}{\Delta}\right| \ll 1$.

\begin{tabular}{|c|c|c|}
\hline & Exact & Approximate \\
\hline & $c_{1}^{2}$ & $2\left(\frac{t}{\Delta}\right)^{2}$ \\
\hline & $c_{2}^{2}$ & 1 \\
\hline & $c_{1} c_{2}$ & $-\sqrt{2} \frac{t}{\Delta}$ \\
\hline$\tilde{\Delta}$ & $\left(c_{2}^{2}-\frac{1}{2} c_{1}^{2}\right) \Delta-3 \sqrt{2} c_{1} c_{2} t$ & $\Delta$ \\
\hline$\tilde{t}_{12}$ & $\frac{1}{2}\left(c_{1}^{2} \Delta+2 \sqrt{2} c_{1} c_{2} t\right)=\frac{1}{\sqrt{2}} t \frac{c_{1}}{c_{2}}$ & $-\frac{t^{2}}{\Delta}$ \\
\hline$\tilde{t}_{1 l}$ & $\left(c_{1}^{2}-c_{2}^{2}\right) t-\frac{1}{\sqrt{2}} c_{1} c_{2} \Delta$ & 0 \\
\hline$\tilde{U}$ & $c_{2}^{2} U+\frac{1}{8} c_{1}^{4}\left(U+2 U^{\prime}\right)$ & $U+\left(\frac{t}{\Delta}\right)^{4} U^{\prime}$ \\
\hline$\tilde{U}^{\prime}$ & $c_{2}^{4} U^{\prime}+\frac{1}{2} c_{1}^{4} U$ & $U^{\prime}+2\left(\frac{t}{\Delta}\right)^{4} U$ \\
\hline$\tilde{K}_{12}$ & $\frac{1}{8} c_{1}^{4}\left(U+2 U^{\prime}\right)$ & $\frac{1}{2}\left(\frac{t}{\Delta}\right)^{4}\left(U+2 U^{\prime}\right)$ \\
\hline$\widetilde{K}_{1 l}$ & $\frac{1}{4} c_{1}^{2}\left[\left(1+c_{2}^{2}\right) U+2 c_{2}^{2} U^{\prime}\right]$ & $\left(\frac{t}{\Delta}\right)^{2}\left(U+U^{\prime}\right)$ \\
\hline$\tilde{J}_{12}$ & $\frac{1}{8} c_{1}^{4}\left(U+2 U^{\prime}\right)$ & $\frac{1}{2}\left(\frac{t}{\Delta}\right)^{4}\left(U+2 U^{\prime}\right)$ \\
\hline$\tilde{J}_{1 l}$ & $\frac{1}{4} c_{1}^{2}\left[\left(1+c_{2}^{2}\right) U+2 c_{2}^{2} U^{\prime}\right]$ & $\left(\frac{t}{\Delta}\right)^{2}\left(U+U^{\prime}\right)$ \\
\hline$\tilde{V}_{1222}$ & $-\frac{1}{4} c_{1}^{2} U+\frac{1}{8} c_{1}^{4}\left(U+2 U^{\prime}\right)$ & $-\frac{1}{2}\left(\frac{t}{\Delta}\right)^{2}\left[U-2\left(\frac{t}{\Delta}\right)^{2} U^{\prime}\right]$ \\
\hline$\tilde{V}_{1 l l l}$ & $\frac{1}{2 \sqrt{2}} c_{1} c_{2}\left(c_{1}^{2} U-2 c_{2}^{2} U^{\prime}\right)$ & $\frac{t}{\Delta}\left[U^{\prime}-\left(\frac{t}{\Delta}\right)^{2} U\right]$ \\
\hline$\tilde{V}_{l 111}$ & $\frac{1}{4 \sqrt{2}} c_{1} c_{2}\left[\left(3+c_{2}^{2}\right) U-2 c_{1}^{2} U^{\prime}\right]$ & $-\frac{t}{\Delta}\left[U-\left(\frac{t}{\Delta}\right)^{2} U^{\prime}\right]$ \\
\hline$\widetilde{V}_{12 l 2}$ & $-\frac{1}{4 \sqrt{2}} c_{1}^{3} c_{2}\left(U+2 U^{\prime}\right)$ & $\frac{1}{2}\left(\frac{t}{\Delta}\right)^{3}\left(U+2 U^{\prime}\right)$ \\
\hline$\tilde{V}_{112 l}$ & $\frac{1}{4} c_{1}^{2}\left(-c_{1}^{2} U+2 c_{2}^{2} U^{\prime}\right)$ & $\left(\frac{t}{\Delta}\right)^{2}\left[U^{\prime}-\left(\frac{t}{\Delta}\right)^{2} U\right]$ \\
\hline$\tilde{V}_{122 l}$ & $-\frac{1}{4 \sqrt{2}} c_{1}^{3} c_{2}\left(U+2 U^{\prime}\right)$ & $\frac{1}{2}\left(\frac{t}{\Delta}\right)^{3}\left(U+2 U^{\prime}\right)$ \\
\hline$\tilde{V}_{1 l l 2}$ & $\frac{1}{4} c_{1}^{2}\left(-c_{1}^{2} U+2 c_{2}^{2} U^{\prime}\right)$ & $\left(\frac{t}{\Delta}\right)^{2}\left[U^{\prime}-\left(\frac{t}{\Delta}\right)^{2} U\right]$ \\
\hline
\end{tabular}

proximation in Eq. (35), we see that such a classification is not possible. Rather, one arrives at Eq. (27) through the inclusion of other Anderson terms, like $\widetilde{J}_{12}$ and $\widetilde{V}_{1222}$, which cancel the erroneous terms in Eq. (31). In this connection, we want to emphasize the importance of the repulsion assisted transfer term $\widetilde{V}_{1222}$ in the "modified" Anderson exchange Hamiltonian $H_{\text {Anderson }}^{\mathrm{C}}$ Consider the signs of $b_{12}$ and $\widetilde{V}_{1222}$. From the exact expression of $\widetilde{V}_{1222}$ in Table I, it can be derived that a necessary condition for $\widetilde{V}_{1222}$ to be positive is
$U^{\prime}>\frac{3}{2} U$. Because repulsion on the metal is always higher than that on the ligand, this condition is never satisfied and consequently $\widetilde{V}_{1222}$ is always negative. At the same time, $b_{12}$ is always positive. ${ }^{32} \widetilde{V}_{1222}$ thus always lowers the magnitude of the conventional Anderson expressions and effectively reduces the condition for validity from $U, U^{\prime} \ll \Delta$ to $U^{\prime} \ll \Delta$, which is more reasonable as it may correspond to some physical reality. Inclusion of $\tilde{V}_{1222}$ in the Anderson theory thus removes the limitation $U \ll \Delta$, which was the main point of critique, eventually leading to the VBCI theory. ${ }^{24} \mathrm{We}$ should note that the modified Anderson exchange $H_{\text {Anderson }}^{\mathrm{C}}$ retains the form of the conventional Anderson exchange $H_{\text {Anderson }}^{\mathrm{B}}$ : by substituting $\beta_{12}=b_{12}+\tilde{V}_{1222}$, we have

$$
H_{\text {Anderson }}^{\mathrm{C}}=\left[-2 \widetilde{J}_{12}+\frac{4 \beta_{12}^{2}}{\tilde{U}-\tilde{K}_{12}}\right] \hat{\mathbf{S}}_{1} \hat{\mathbf{S}}_{2},
$$

where we must keep in mind that $\beta_{12}$ can be reduced considerably with respect to $b_{12}$. These findings are visualized in Fig. 3, where the three Anderson-based approximations are plotted together with the exact exchange. The plot clearly shows that the conventional approximations $H_{\text {Anderson }}^{\mathrm{A}}$ and $H_{\text {Anderson }}^{\mathrm{B}}$ calculate the exchange splitting much too high, independent of $t$ and $U^{\prime}$. The modified form $H_{\text {Anderson }}^{\mathrm{C}}$ corrects this overestimation and gives good agreement with the exact exchange, even for $U^{\prime} \approx \Delta$. Hence, the usual assumption that $\widetilde{V}_{1222}$ can be neglected ${ }^{31}$ is not justified.

An interesting question is how variations of the system parameters affect the validity of the approaches. Consider, for example, the repulsion $U$ on the metal ion. In the classical Anderson theory [Eqs. (31) and (32)], a hypothetical increase of $U$, at fixed $\Delta$, would increase the exchange interaction, going to infinity for $U \rightarrow \infty .{ }^{33}$ From the VBCI theory, however, we know that in the case of infinite Coulomb repulsion on the metal, a source of finite exchange is present in the form of a double ligand-to-metal excitation. In principle, one might think that this effect could also be recovered in the Anderson theory by including the ligand orbital $\Psi_{1+}$ in the perturbational calculation. This has indeed shown to successfully reproduce the VBCI expression: Eq. (34). However, when $U$ grows further, some approximations used there are not valid anymore. It turns out that the Anderson orbitals do not provide a useful starting point for perturbation theory anymore, as can be seen in Fig. 4, where the complete second-, third-, and fourth-order perturbational approximations are plotted. The reason for this behavior is to be found in the composition of the Anderson orbitals. Each magnetic orbital contains a contribution on its neighboring metal site [see Eqs. (21) and (22)]. One naturally expects this contribution to diminish with increasing $U$ so as to prevent two electrons from residing on the same metal site when their spins are paired. However, this does not happen with the Anderson orbitals: in Sec. III A, it was shown that they depend only on $\frac{t}{\Delta}$. Thus, a variation of $U$ does not alter the orbitals at all. As a result, the first-order energy of the singlet state increases with $U$, while that of the triplet state is unaffected. Eventually, this wrong-signed splitting reaches a point where perturbation theory cannot recover this anymore, as is apparent 


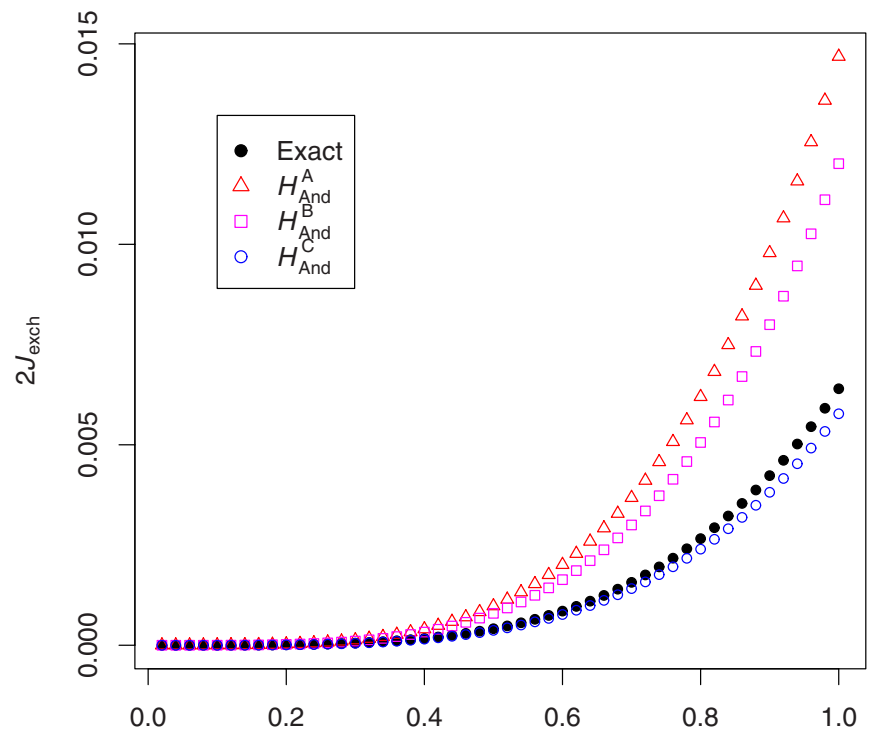

(a)

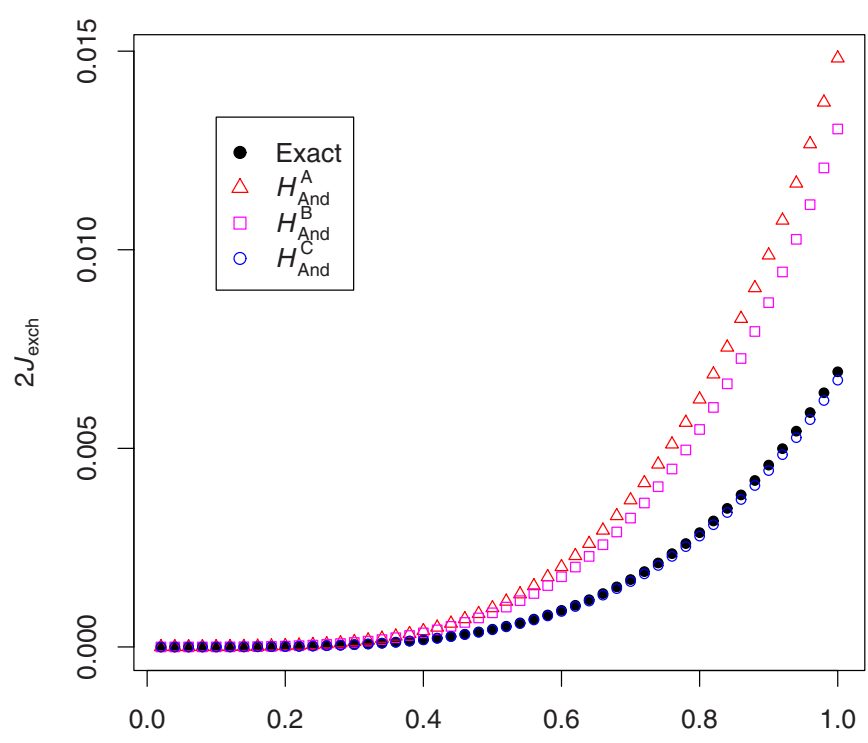

(b)

FIG. 3. (Color online) Exchange splittings as a function of $t$. The three Anderson-based approaches are compared with the exact solution. (a) $\Delta=10, U=10, U^{\prime}=10$; (b) $\Delta=10, U=10, U^{\prime}=5$.

in Fig. 4. Note that the first-order splitting equals $-2 \widetilde{J}_{12}$ [Eq. (24)] so that the discussed effect of $U$ is totally contained in the exact expression of $\tilde{J}_{12}$ (Table I). In general, it is not possible for the neighboring-ion contribution to vanish while still preserving the delocalization on the ligand because of the orthogonality of the magnetic orbitals: the contribution on the neighboring ion is essential to keep them orthogonal. Despite the fact that the Anderson orbitals are inappropriate for large $U$, the modified Anderson exchange $H_{\text {Anderson }}^{\mathrm{C}}$ still reproduces the exact exchange quite well (Fig. 4) because the approximation in Eq. (33) remains valid.

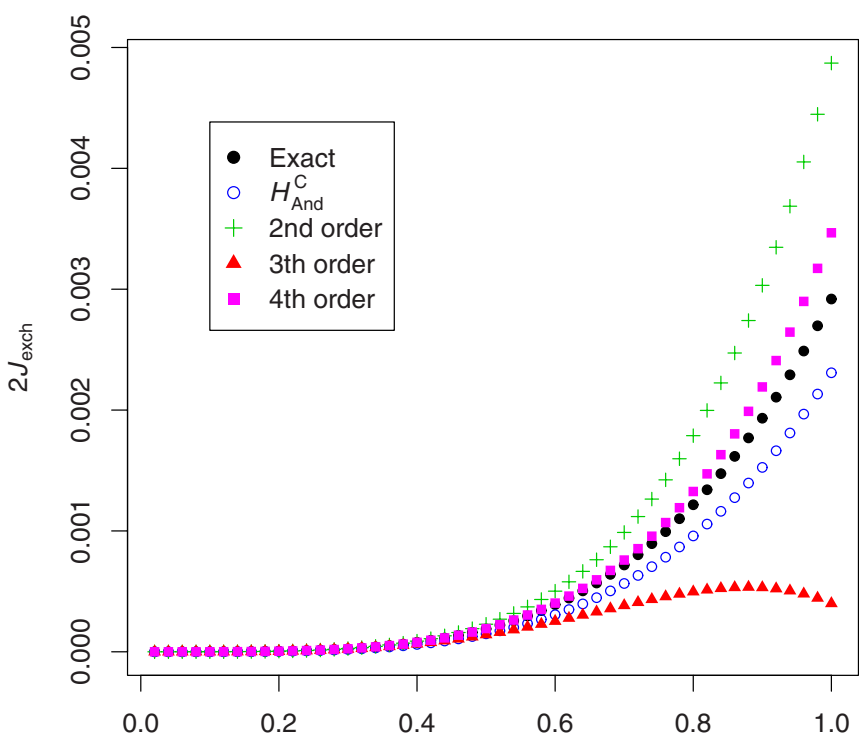

(a)

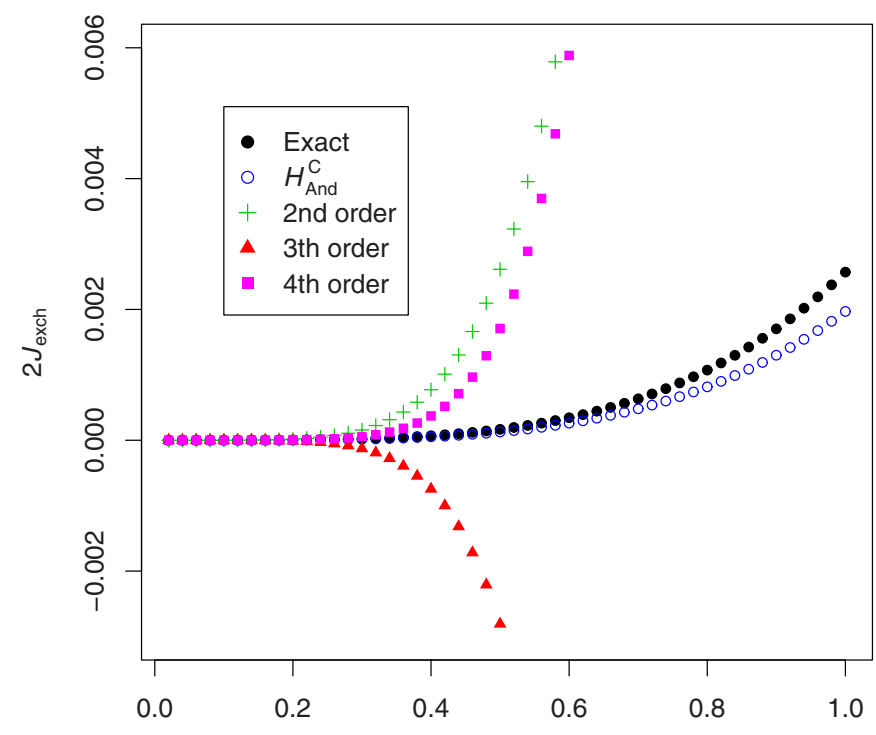

(b)

FIG. 4. (Color online) Exchange splittings as a function of $t$ for increased values of $U$. The modified Anderson exchange and complete second-, third-, and fourth-order perturbational approximations are compared with the exact solution. (a) $\Delta=10, U=100, U^{\prime}$ =10; (b) $\Delta=10, U=1000, U^{\prime}=10$.

\section{Cases for which $\left|\frac{t}{\Delta}\right| \ll 1$ is not valid}

As was repeatedly pointed out, ${ }^{24,26,30,34}$ the assumption $\left|\frac{t}{\Delta}\right| \ll 1$ is not generally valid. In fact, numerous examples exist where the ligand-metal energy gap $\Delta$ is known to be quite small or the metal-ligand hybridization element $t$ to be rather large. In these cases, we cannot rely on a Taylor series approximation of the orbital coefficients $c_{1}$ and $c_{2}$, as was done in Eqs. (31)-(34). Instead, information will be inferred from plots.

In the previous section, we saw that the conventional Anderson approaches [Eqs. (28) and (29)] always gave too 


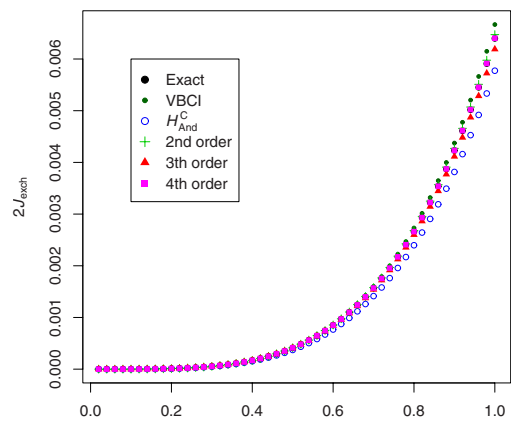

(a)
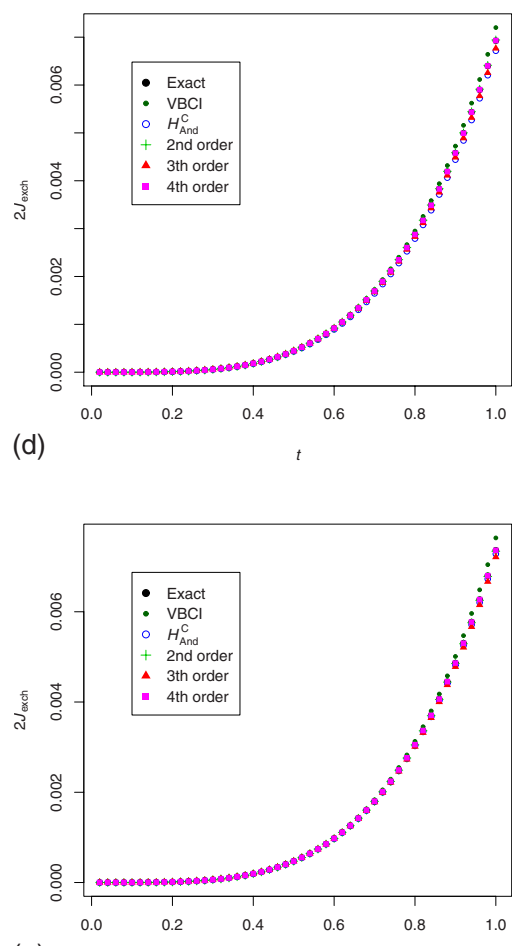

(g)

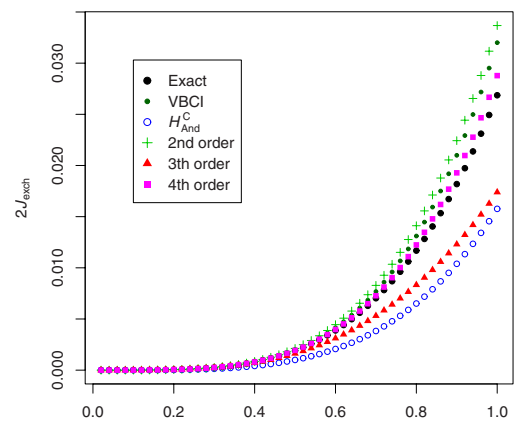

(b)

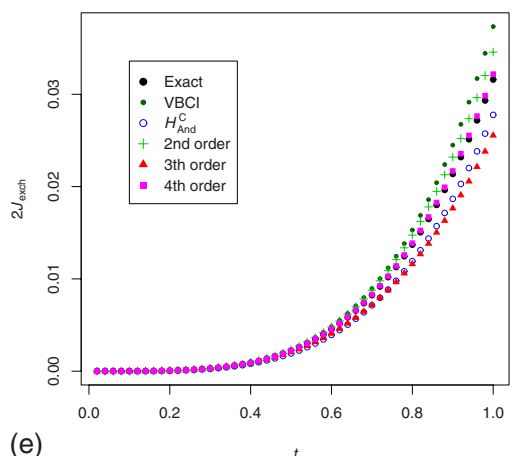

(e)

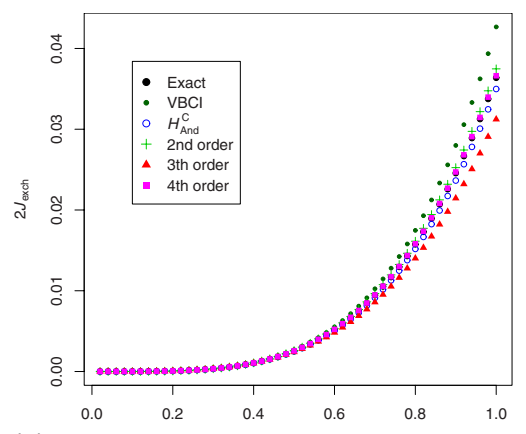

(h)

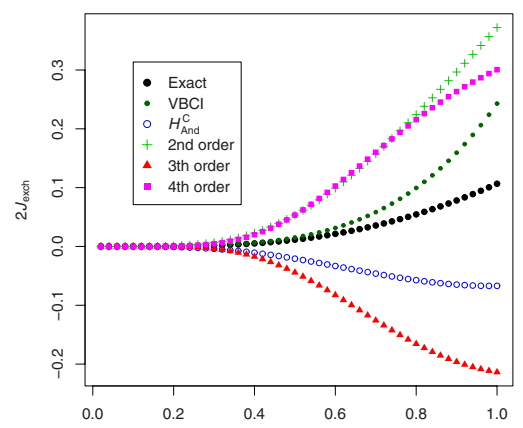

(c)
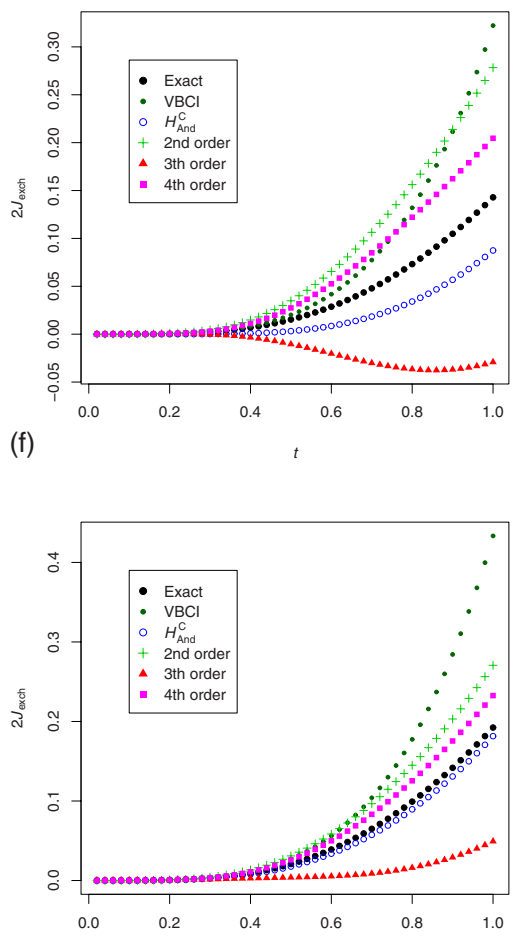

(i)

FIG. 5. (Color online) Exchange splittings as a function of $t$. The modified Anderson exchange and complete second-, third-, and fourth-order perturbational approximations are compared with VBCI and the exact solution. Values of $\Delta$ are varied per column. Values of $U^{\prime}$ are varied per row. (a) $\Delta=10, U=10, U^{\prime}=10$; (b) $\Delta=5, U=10, U^{\prime}=10$; (c) $\Delta=2, U=10, U^{\prime}=10$; (d) $\Delta=10, U=10, U^{\prime}=5$; (e) $\Delta=5$, $U=10, U^{\prime}=5$; (f) $\Delta=2, U=10, U^{\prime}=5$; (g) $\Delta=10, U=10, U^{\prime}=2$; (h) $\Delta=5, U=10, U^{\prime}=2$; (i) $\Delta=2, U=10, U^{\prime}=2$

high exchange interactions when $U \geqslant \Delta$. This situation is left unchanged for what is to come and we shall therefore not consider these approaches anymore, but focus on the VBCI exchange, the modified Anderson exchange $H_{\text {Anderson }}^{\mathrm{C}}$, and the Anderson-based perturbations up to fourth order. Both an increase of $t$ and a decrease of $\Delta$ will be examined as well as the effect of varying $U^{\prime}$, since the Coulomb repulsion on the ligand can vary considerably according to the ligand's nature, though it will generally be smaller than $U$, which will be held constant as a reference energy.

A first series of plots is presented in Fig. 5, where $\Delta$ is lowered from left to right while $U^{\prime}$ is lowered from top to bottom. In the left column of Fig. 5, all approaches reproduce the exchange splitting very well because the conditions correspond with those discussed in the previous section: $\left|\frac{t}{\Delta}\right| \ll 1, U$ and $\Delta$ are of the same order of magnitude, and $U^{\prime}$ is of the same order of magnitude as or smaller than $\Delta$. In the middle and the right columns of Fig. 5, the first condition is gradually less obeyed as $\Delta$ decreases. A first observation concerns the VBCI approach. As $\Delta$ decreases, the VBCI exchange behaves gradually more erroneously, calculating the exchange energy too high, obviously due to its perturbative nature with respect to $\left|\frac{t}{\Delta}\right|$. Note that the failure of VBCI is solely due to the increase of $\left|\frac{t}{\Delta}\right|$, independent of the value of $U^{\prime}{ }^{35}$ This situation is different for the Anderson approaches: here, a lowering of $\Delta$ also has a negative influence on the quality of the approximation, as is most apparent in the first row of Fig. 5. However, lowering $U^{\prime}$ brings these approximations again closer to the exact solution. Consider, for example, the third column of Fig. 5, where $\Delta=2$. When $U^{\prime}$ $=10$, there is no agreement at all, but on diminishing $U^{\prime}$ to 5 and 2, the Anderson approaches tend to get better, and curi- 


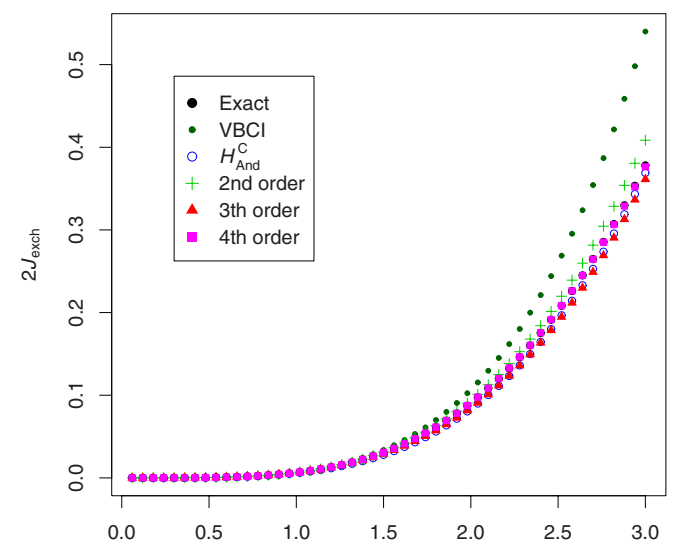

(a)

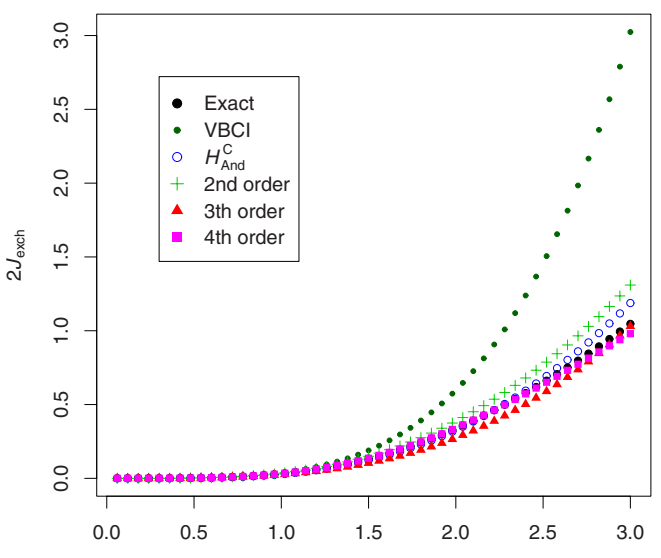

(b)

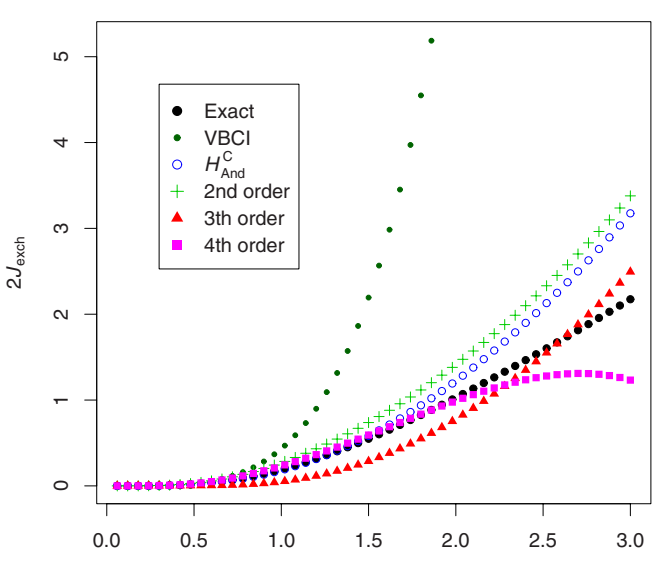

(c)

FIG. 6. (Color online) Exchange splittings as a function of $t$. The modified Anderson exchange and complete second-, third-, and fourth-order perturbational approximations are compared with VBCI and the exact solution, for extended values of $t$. (a) $\Delta=10$, $U=10, U^{\prime}=10$; (b) $\Delta=5, U=10, U^{\prime}=5$; (c) $\Delta=2, U=10, U^{\prime}=2$.

ously, it is the modified Anderson exchange $H_{\text {Anderson }}^{\mathrm{C}}$ which in the end gives closest correspondence to the exact solution: Fig. 5(i). One notices that even then, higher-order corrections do not tend to converge to the exact solution, so that the success of $H_{\text {Anderson }}^{\mathrm{C}}$ is rather remarkable. This case resembles in a sense the one discussed in the previous section, where an increase of $U$ was found to have a dramatic effect on the quality of the starting point configurations for perturbation theory in the Anderson approaches. Here again, only $\frac{t}{\Delta}$ determines the composition of the orbitals. When $\left|\frac{t}{\Delta}\right|$ increases, the magnetic orbitals get more delocalized onto the ligand and the neighboring metal. In the singlet state then, the wave function contains a contribution with the two holes paired on the ligand. If $U^{\prime}$ is relatively high, this contribution may increase the energy of this state to such an extent that perturbation theory is not capable anymore of correcting this. This analysis in terms of contributing configurations in the atomic basis essentially comes down to stating that the first order exchange splitting, $-2 \widetilde{J}_{12}$, or the potential exchange, increases as $\left|\frac{t}{\Delta}\right|$ and $U^{\prime}$ (and $U$ ) increase (see the exact expression for $\widetilde{J}_{12}$ in Table I), placing the triplet state at the lowest energy, an error that must be corrected in the subsequent perturbational orders.

In this connection, we would like to mention that an unrestricted Hartree-Fock (UHF) calculation of the exchange energy in this model will also fail for relatively large $U^{\prime}$. With UHF, the orbitals are separately optimized for the $M_{S}$ $=1$ and the broken symmetry $M_{S}=0$ state. In the limit of large $U^{\prime}$, the magnetic orbitals of the latter state will be the pure atomic starting orbitals on the metals, i.e., without any delocalization on the ligand. The $M_{S}=0$ state will therefore not gain energy. The solution for the $M_{S}=1$ state, on the other hand, will be the same as the HF solution for this state, given in Sec. III A. Through bonding with the ligand, it will gain energy. The UHF calculation will therefore predict a ferromagnetic ground state in this case.

In Fig. 6, a series of plots is shown where the maximum value of $t$ is set to 3 . These are just extensions of the plots in Figs. 5(a), 5(e), and 5(i). Again, we see that the VBCI curve is always too high, again due to the increase of $\left|\frac{t}{\Delta}\right|$. On the other hand, the Anderson approach behaves very well in Figs. 6(a) and 6(b), where especially the modified Anderson exchange $H_{\text {Anderson }}^{\mathrm{C}}$ is seen to nearly perfectly reproduce the exact exchange. Only in an extreme situation as in Fig. 6(c), the latter also fails, although it is still much better than VBCI. Clearly, the quality of the Anderson exchange is much less sensitive to an increase of $t$ than to a decrease of $\Delta$.

The previous examples, where the condition $\left|\frac{t}{\Delta}\right| \ll 1$ is not fulfilled, show the importance of a nonperturbative incorporation of the metal-ligand covalency, as in the Anderson HF orbitals, as opposed to a perturbative approach such as VBCI. Still, there remain cases in which neither VBCI nor Anderson provides a satisfactory solution to the exchange problem.

\section{EXACT TREATMENT OF THE METAL-LIGAND COVALENCY}

A physical theory that is to provide an insight into the origin of the magnetic interaction between centra should ideally define a starting point where all important physical interactions are included except for those that differentiate between the relative spin direction of the magnetic electrons. The important effect to incorporate is the delocalization of 
each magnetic electron onto its neighboring ligand, an effect that is since long known to be essential for superexchange to occur. The purpose of the Anderson orbitals is indeed to incorporate this covalency via a HF-type calculation. The choice of the ferromagnetic configuration as substrate for the $\mathrm{HF}$ calculation, as was done in Sec. III A, ensures that no exchange effects are included in the construction of the orbitals, because unpaired spins cannot reside on the same metal or ligand orbital. However, when these orbitals are used to construct the antiferromagnetic ground state, pairing of spins on the sites is allowed. As a result, the equivalence of starting points for the ferro- and antiferromagnetic states is broken. Under certain conditions, this may lead to a failure of the Anderson exchange, as discussed in the previous section. The VBCI approach treats the ferro- and antiferromagnetic states on equal footing in including the metal-ligand covalency but does so in a perturbative way. This works well if $\left|\frac{t}{\Delta}\right| \ll 1$ but inevitably leads to errors when this condition is not fulfilled.

Within the present model, it is possible to obtain an exchange expression satisfying both requirements: metal-ligand covalency is treated exactly and magnetic exchange occurs in second order of perturbation. We start from the Hamiltonian matrices in the atomic basis [Eqs. (10) and (11)] and notice that the parts describing the interaction of the "ground" covalent state $\left|\operatorname{cov} ; S M_{S}\right\rangle$ [Eqs. (4) and (8)], where each metal center is occupied by one hole, with the state $\left|\operatorname{lm} ; S M_{S}\right\rangle$ [Eqs. (5) and (9)], where one hole is excited to the ligand site, are equal:

$$
\left(\begin{array}{cc}
0 & -\sqrt{2} t \\
& \Delta
\end{array}\right)
$$

On diagonalizing this matrix, we obtain an exact inclusion of covalency that is equal for the triplet and singlet states. Note that this matrix is the same as the HF matrix [Eq. (15)] so that the solutions of the latter can be transferred to the present problem. The eigenstates are then

$$
\begin{aligned}
& \left|A ; S M_{S}\right\rangle=c_{2}\left|\operatorname{cov} ; S M_{S}\right\rangle-c_{1}\left|l m ; S M_{S}\right\rangle, \\
& \left|B ; S M_{S}\right\rangle=c_{1}\left|\operatorname{cov} ; S M_{S}\right\rangle+c_{2}\left|l m ; S M_{S}\right\rangle,
\end{aligned}
$$

and the transformed Hamiltonian matrices,

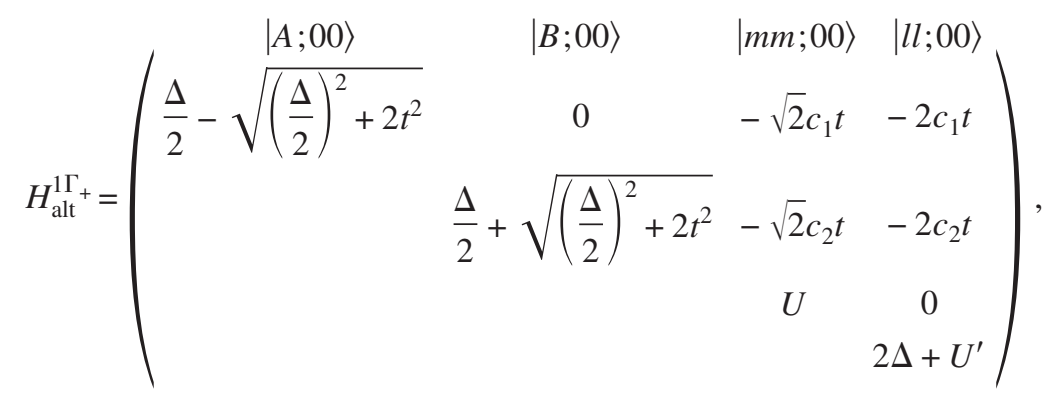

$$
H_{\text {alt }}^{3 \Gamma_{-}}=\left(\begin{array}{cc}
|A ; 11\rangle & |B ; 11\rangle \\
\frac{\Delta}{2}-\sqrt{\left(\frac{\Delta}{2}\right)^{2}+2 t^{2}} & 0 \\
& \frac{\Delta}{2}+\sqrt{\left(\frac{\Delta}{2}\right)^{2}+2 t^{2}}
\end{array}\right),
$$

with $c_{1}$ and $c_{2}$ as in Eqs. (19) and (20). The singlet and triplet ground states have the same starting point energy: $\frac{\Delta}{2}$ $-\sqrt{\left(\frac{\Delta}{2}\right)^{2}+2 t^{2}}$. With $\left|A ; S M_{S}\right\rangle$, we have constructed a state in which the holes are delocalized over the metal and ligand sites but are never allowed to pair on the same site. It is thus a purely covalent starting point. Note that for the triplet state, this starting point is the same as the one obtained in the Anderson HF calculation. Indeed, parallel spins automatically exclude each other from the same site. For the singlet state, on the other hand, the present starting point cannot be described as a single configuration. As a result of the trans- formation, the ground state of $H_{\text {alt }}^{1} \Gamma^{+}$has now a direct matrix element with the third and fourth basis states, viz., $|\mathrm{mm} ; 00\rangle$ and $|l l ; 00\rangle$. These interactions are absent in $H_{\text {alt }}^{\Gamma_{-}}$and thus provide the path for exchange interaction, which is already obtained in second order of perturbation:

$$
\begin{aligned}
H_{\text {alt }}^{\text {eff }}= & 2 t^{2} c_{1}^{2}\left(\frac{1}{U-\left(\frac{\Delta}{2}-\sqrt{\left(\frac{\Delta}{2}\right)^{2}+2 t^{2}}\right)}\right. \\
& \left.+\frac{2}{2 \Delta+U^{\prime}-\left(\frac{\Delta}{2}-\sqrt{\left(\frac{\Delta}{2}\right)^{2}+2 t^{2}}\right)}\right) \hat{\mathbf{S}}_{1} \hat{\mathbf{S}}_{2} \\
= & 2 t^{2} c_{1}^{2}\left(\frac{1}{U-\sqrt{2} t \frac{c_{1}}{c_{2}}}+\frac{2}{2 \Delta+U^{\prime}-\sqrt{2} t \frac{c_{1}}{c_{2}}}\right) \hat{\mathbf{S}}_{1} \hat{\mathbf{S}}_{2} .
\end{aligned}
$$

This equation is very similar to the VBCI equation [Eq. (27)] 


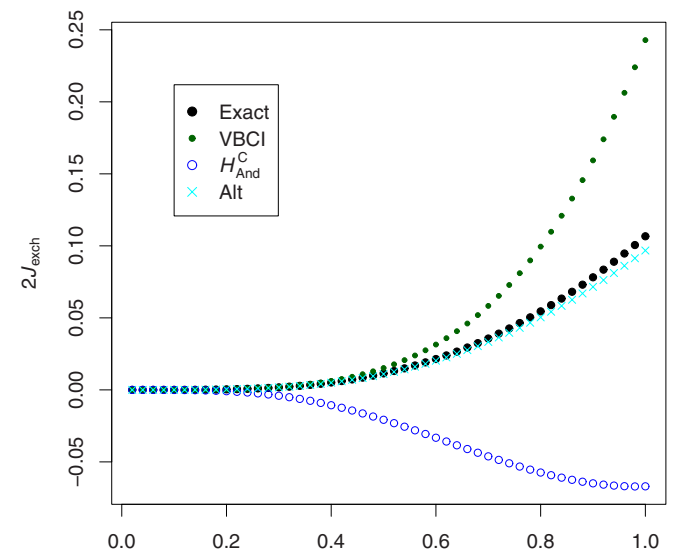

(a)

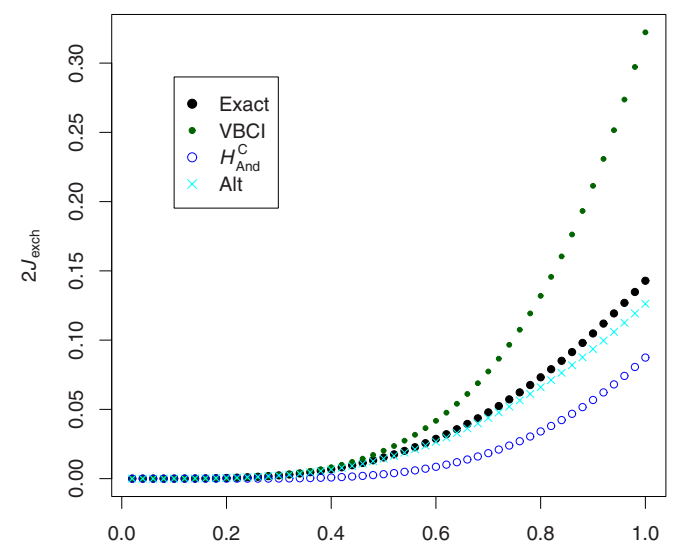

(b)

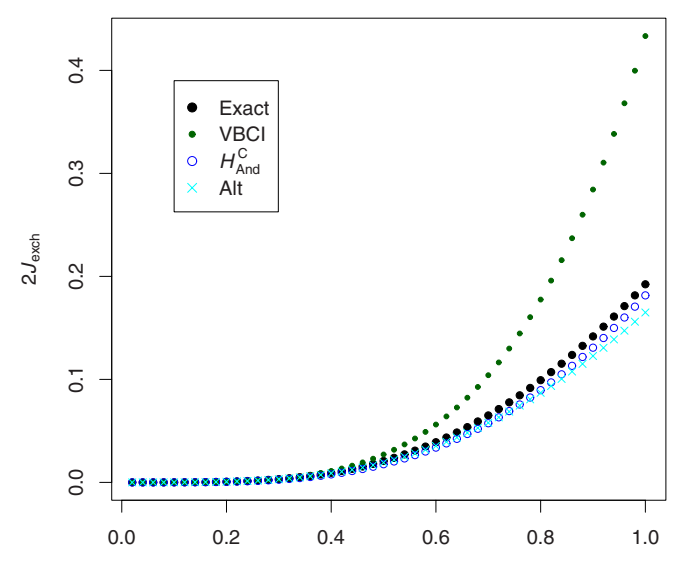

(c)

FIG. 7. (Color online) Exchange splittings as a function of $t$. The modified Anderson exchange and VBCI are compared with the alternative exchange and the exact solution. (a) $\Delta=2, U=10, U^{\prime}$ $=10 ; \quad$ (b) $\quad \Delta=2, \quad U=10, \quad U^{\prime}=5 ; \quad$ (c) $\quad \Delta=2, \quad U=10$, $U^{\prime}=2$.

and reduces to the latter if $\left|\frac{t}{\Delta}\right| \ll 1$ and $\left|\frac{t}{U}\right| \ll 1$. $H_{\text {alt }}^{\text {eff }}$ is, however, not restricted by a limitation on the value of $\left|\frac{t}{\Delta}\right|$, as was the VBCI equation, nor will it fail when $U$ or $U^{\prime}$ are too large, as was the case with the Anderson approach. Figures 7 and 8 present some plots of conditions that were found to be

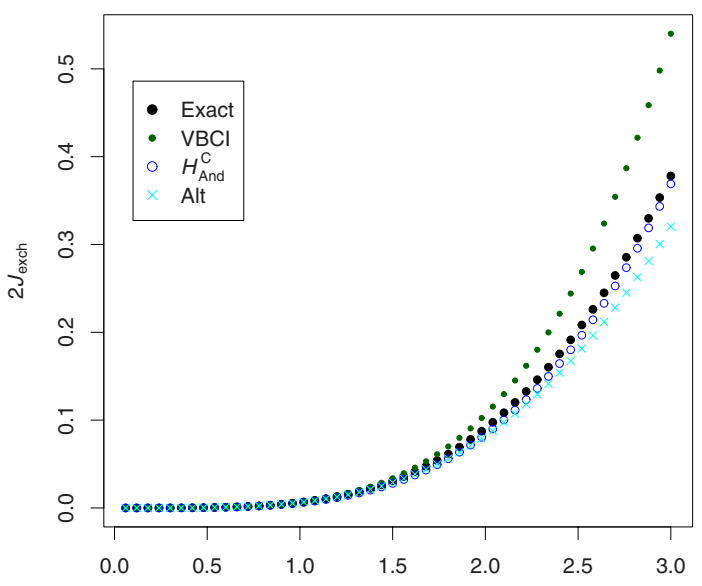

(a)

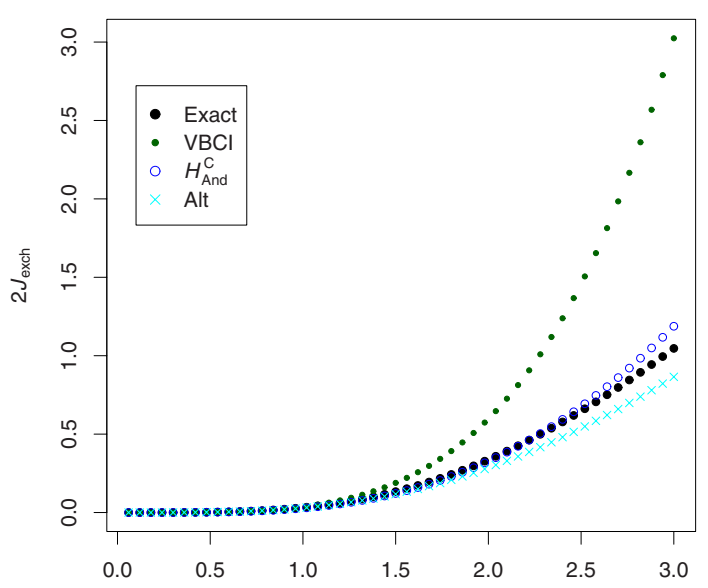

(b)

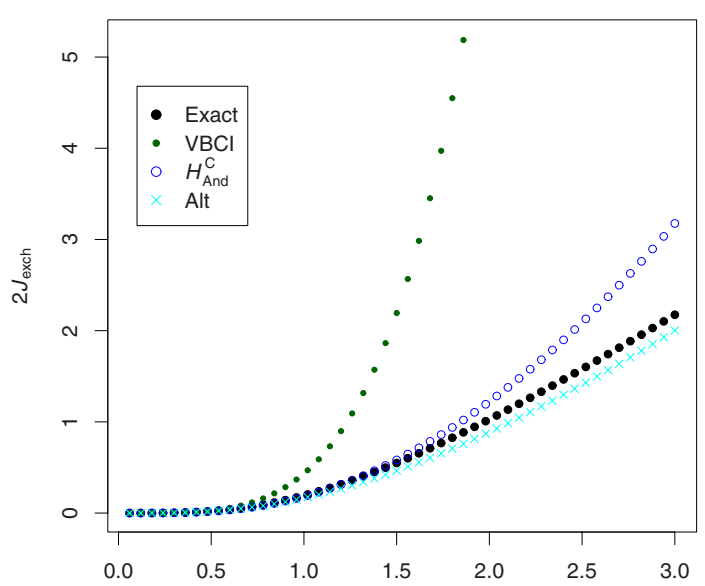

(c)

FIG. 8. (Color online) Exchange splittings as a function of $t$. The modified Anderson exchange and VBCI are compared with the alternative exchange and the exact solution, for extended values of t. (a) $\Delta=10, U=10, U^{\prime}=10$; (b) $\Delta=5, U=10, U^{\prime}=5$; (c) $\Delta=2$, $U=10, U^{\prime}=2$.

problematic for VBCI, Anderson, or both. The present approach has been included, in comparison with VBCI, the modified Anderson, and the exact solution. The plots in Fig. 
7 represent the case of small $\Delta$ and correspond to the third column of Fig. 5. Especially in Fig. 7(a), the present approach is seen to be capable of dealing with a situation in which VBCI and Anderson are unsuccessful. Only in Fig. 7(c) the modified Anderson exchange comes closer to the exact solution. Larger values of $t$ are considered in Fig. 8 with plots corresponding to Figs. 6(a)-6(c). Overall, the present approach proves to be versatile in treating the exchange problem in a broad range of situations.

How stable is the second-order perturbational expression [Eq. (42)]? In analogy with VBCI, one might expect that it would diverge for large $t$ or small $U, U^{\prime}$, or $\Delta$. This is, however, not the case; it can be shown that the perturbational fractions are, in absolute value, always smaller than 1:

$$
\begin{gathered}
\frac{\sqrt{2}\left|c_{1} t\right|}{U-\sqrt{2} t \frac{c_{1}}{c_{2}}}<1, \\
\frac{2\left|c_{1} t\right|}{2 \Delta+U^{\prime}-\sqrt{2} t \frac{c_{1}}{c_{2}}}<1,
\end{gathered}
$$

for $\Delta, U, U^{\prime},|t|>0$. This means that the perturbational expansion is always valid and explains why, for example, this second-order exchange expression does never diverge from the exact solution, as can be clearly seen in Fig. 8(c).

\section{CONCLUSION}

We have investigated the application of the Anderson and the VBCI theories to a basic exchange model, in which the ground state is always antiferromagnetic. The Anderson orbitals can be obtained exactly, allowing us to compare both methods quantitatively. A first, and remarkable, observation is that the original Anderson exchange expression, including only the kinetic transfer $b_{12}$ and the potential exchange $\widetilde{J}_{12}$, is not an accurate approximation if the rather unphysical condition $U \ll \Delta$ is not fulfilled, even in the most well-behaved situations (Fig. 3). The reason is that the often disregarded "repulsion assisted transfer" term $\widetilde{V}_{1222}$ is not negligible with respect to $b_{12}$. Inclusion of the former indeed removes the mentioned restricting condition, while the form of the original Anderson expression is preserved: Eq. (36). We have discussed the importance of the on-site metal and ligand Coulomb repulsions $U$ and $U^{\prime}$ for the reliability of the Anderson method. The composition of the Anderson orbitals does not depend on $U$ or $U^{\prime}$. For relatively high values of these parameters, the Anderson ground configurations are not valid starting points for perturbation theory anymore because the first-order energy of the singlet state is raised too much above the triplet energy. The VBCI method is reliable for systems with weak metal-ligand covalency, i.e., $\left|\frac{t}{\Delta}\right| \ll 1$. Whenever this is not the case, it fails due to its perturbational description of covalency. In some of these cases, the Anderson method, incorporating the metal-ligand covalency in a variational way in its orbitals, may prove more successful, although this again depends on the relative magnitude of $U$ and $U^{\prime}$. To overcome the problems of both methods, we have suggested that the metal-ligand covalency be incorporated exactly in the starting point wave functions followed by a second-order evaluation of the exchange effects. This way, we obtained an exchange expression that does not suffer from any restricting condition on the model parameters.

\section{ACKNOWLEDGMENT}

W.V.d.H. (Aspirant van het FWO) gratefully acknowledges financial support from the Flemish Science Foundation (FWO).

\section{APPENDIX A: DERIVATION OF THE HARTREE-FOCK ORBITALS}

It is not immediately obvious that a HF treatment of the triplet state in the hole representation is completely equivalent to a ROHF treatment of the same state in the electron representation. In the electron case, a restriction is imposed upon the doubly occupied orbital, namely, that it has the same spatial part for both spin up and spin down. In the hole case, on the other hand, there are no paired holes and a similar restriction seems to be absent. In the following, it will be explicitly shown that the obtained HF orbitals are nevertheless identical.

The ground ${ }^{3} \Gamma_{-}$state is written in the electron representation:

$$
|\operatorname{cov} ; 11\rangle=\left|\Psi_{2+} \Psi_{-} \Psi_{1+} \bar{\Psi}_{1+}\right| .
$$

Symmetry restrictions and mutual orthonormality require the molecular orbitals to be of the form

$$
\Psi_{1+}=a_{1} \phi_{+}+a_{2} \phi_{l}, \quad \Psi_{-}=\phi_{-}, \quad \Psi_{2+}=a_{2} \phi_{+}-a_{1} \phi_{l},
$$

with $a_{1}^{2}+a_{2}^{2}=1$. Finding the HF orbitals means minimizing the energy of $|\operatorname{cov} ; 11\rangle$ with respect to $a_{1}$ and $a_{2}$. Using Hamiltonian (1) for electrons and the identity $a_{1}^{2}+a_{2}^{2}=1$, we find

$$
\begin{aligned}
E= & \langle\operatorname{cov} ; 11|H| \operatorname{cov} ; 11\rangle=2 h_{11}+h_{l l}+a_{1}^{2} h_{11}+a_{2}^{2} h_{l l} \\
& +2 \sqrt{2} a_{1} a_{2} t+a_{1}^{2} U+a_{2}^{2} U^{\prime} .
\end{aligned}
$$

Stationary points on the unit circle $\left(a_{1}^{2}+a_{2}^{2}=1\right)$ obey the equations

$$
\frac{\partial E}{\partial a_{i}}-\lambda \frac{\partial}{\partial a_{i}}\left(a_{1}^{2}+a_{2}^{2}\right)=0 \quad \text { with } i=1,2 .
$$

Applying Eq. (A4) to Eq. (A3) gives

$$
\left(\begin{array}{cc}
h_{11}+U-\lambda & \sqrt{2} t \\
\sqrt{2} t & h_{l l}+U^{\prime}-\lambda
\end{array}\right)\left(\begin{array}{l}
a_{1} \\
a_{2}
\end{array}\right)=\left(\begin{array}{l}
0 \\
0
\end{array}\right),
$$

or, setting $\lambda^{\prime}=-\lambda+h_{11}+U$, using $\Delta=h_{11}+U-h_{l l}-U^{\prime}$, and changing the overall sign,

$$
\left(\begin{array}{cc}
-\lambda^{\prime} & -\sqrt{2} t \\
-\sqrt{2} t & \Delta-\lambda^{\prime}
\end{array}\right)\left(\begin{array}{l}
a_{1} \\
a_{2}
\end{array}\right)=\left(\begin{array}{l}
0 \\
0
\end{array}\right) .
$$

This eigenvalue equation is exactly the same as the eigenvalue equation of the HF matrix for the holes [Eq. (15)]. 
There are thus two solutions: $\left(a_{1}, a_{2}, \lambda^{\prime}\right)=\left(c_{1}, c_{2},-\sqrt{2} t \frac{c_{2}}{c_{1}}\right)$ and $\left(a_{1}, a_{2}, \lambda^{\prime}\right)=\left(c_{2},-c_{1}, \sqrt{2} t \frac{c_{1}}{c_{2}}\right)$, with $c_{1}$ and $c_{2}$ as in Eqs. (19) and (20). To determine which one corresponds to the lowest energy, we multiply Eq. (A5) in front with $\left(a_{1} a_{2}\right)$ and get

$$
a_{1}^{2} h_{11}+a_{1}^{2} U+a_{2}^{2} h_{22}+a_{2}^{2} U^{\prime}+2 \sqrt{2} a_{1} a_{2} t=\lambda .
$$

Using Eq. (A3), this gives

$$
E=2 h_{11}+h_{l l}+\lambda=3 h_{11}+h_{l l}+U-\lambda^{\prime} .
$$

From Eq. (A8) and the fact that $t \frac{c_{2}}{c_{1}}$ is always negative, we conclude that the solution $\left(a_{1}, a_{2}, \lambda^{\prime}\right)=\left(c_{1}, c_{2},-\sqrt{2} t \frac{c_{2}}{c_{1}}\right)$ corresponds to the sought energy minimum of $|\operatorname{cov} ; 11\rangle$. Thus, the ROHF molecular orbitals in Eq. (A2) are exactly equal to the HF orbitals in Eqs. (16)-(18), found for the hole representation.

\section{APPENDIX B: HAMILTONIAN FOR HOLES IN AN ANDERSON-LIKE BASIS $\{1,2, l\}$}

If an orthogonal transformation of the (real) orbitals of a general Hamiltonian

$$
H=\sum_{i, j, \sigma} t_{i j} a_{i \sigma}^{\dagger} a_{j \sigma}+\frac{1}{2} \sum_{i, j, k, l} V_{i j k l} \sum_{\sigma, \sigma^{\prime}} a_{i \sigma}^{\dagger} a_{j \sigma^{\prime}}^{\dagger} a_{l \sigma^{\prime}} a_{k \sigma}
$$

is applied, with $b_{p}=\sum_{i} c_{i p} a_{i}$ and $b_{p}^{\dagger}=\sum_{i} c_{i p} a_{i}^{\dagger}$, the Hamiltonian (B1) transforms to

$$
H=\sum_{p, q, \sigma} \tilde{t}_{p q} b_{p \sigma}^{\dagger} b_{q \sigma}+\frac{1}{2} \sum_{p, q, r, s} \tilde{V}_{p q r s} \sum_{\sigma, \sigma^{\prime}} b_{p \sigma}^{\dagger} b_{q \sigma^{\prime}}^{\dagger} b_{s \sigma^{\prime}} b_{r \sigma}
$$

where

$$
\tilde{t}_{p q}=\sum_{i, j} c_{i p} c_{j q} t_{i j}, \quad \tilde{V}_{p q r s}=\sum_{i, j, k, l} c_{i p} c_{j q} c_{k r} c_{l s} V_{i j k l}
$$

are the matrix elements in the new basis. For a general set of three localized orbitals in the present model, and thus, in particular, for the Anderson orbitals, the Hamiltonian (3) can therefore be written as

$$
\begin{aligned}
& H^{\text {holes }}=\sum_{\sigma}\left[\widetilde{\Delta} n_{l \sigma}+\frac{1}{2} \widetilde{U}^{\prime} n_{l \sigma} n_{l-\sigma}+\frac{1}{2} \widetilde{U}\left(n_{1 \sigma} n_{1-\sigma}+n_{2 \sigma^{2}} n_{2-\sigma}\right)\right]+\sum_{\sigma, \sigma^{\prime}}\left[\widetilde{K}_{1 l}\left(n_{1 \sigma} n_{l \sigma^{\prime}}+n_{2 \sigma} n_{l \sigma^{\prime}}\right)+\widetilde{K}_{12} n_{1 \sigma} n_{2 \sigma^{\prime}}\right] \\
& +\sum_{\sigma}\left[\widetilde{t}_{1 l}\left(b_{1 \sigma}^{\dagger} b_{l \sigma}+b_{l \sigma}^{\dagger} b_{1 \sigma}+b_{2 \sigma^{\prime}}^{\dagger} b_{l \sigma}+b_{l \sigma}^{\dagger} b_{2 \sigma}\right)+\widetilde{t}_{12}\left(b_{1 \sigma}^{\dagger} b_{2 \sigma}+b_{2 \sigma}^{\dagger} b_{1 \sigma}\right)\right]+\sum_{\sigma, \sigma^{\prime}}\left[-\widetilde{J}_{1 l}\left(b_{1 \sigma}^{\dagger} b_{1 \sigma^{\prime}} b_{l \sigma^{\prime}}^{\dagger} b_{l \sigma}+b_{2 \sigma^{\prime}}^{\dagger} b_{2 \sigma^{\prime}} b_{l \sigma^{\prime}}^{\dagger} b_{l \sigma}\right)\right. \\
& \left.-\widetilde{J}_{12} b_{1 \sigma}^{\dagger} b_{1 \sigma^{\prime}} b_{2 \sigma^{\prime}}^{\dagger} b_{2 \sigma}\right]+\widetilde{V}_{11 l l}\left(b_{1 \sigma}^{\dagger} b_{1-\sigma}^{\dagger} b_{l-\sigma} b_{l \sigma}+b_{2 \sigma}^{\dagger} b_{2-\sigma}^{\dagger} b_{l-\sigma} b_{l \sigma}+b_{l \sigma}^{\dagger} b_{l-\sigma}^{\dagger} b_{1-\sigma} b_{1 \sigma}+b_{l \sigma}^{\dagger} b_{l-\sigma}^{\dagger} b_{2-\sigma} b_{2 \sigma}\right)+\widetilde{V}_{1122}\left(b_{1 \sigma}^{\dagger} b_{1-\sigma}^{\dagger} b_{2-\sigma} b_{2 \sigma}\right. \\
& \left.+b_{2 \sigma}^{\dagger} b_{2-\sigma}^{\dagger} b_{1-\sigma} b_{1 \sigma}\right)+\sum_{\sigma}\left\{\left(b_{1 \sigma}^{\dagger} b_{l \sigma}+b_{l \sigma}^{\dagger} b_{1 \sigma}\right)\left(\tilde{V}_{1 l l l} n_{l-\sigma}+\widetilde{V}_{l 111} n_{1-\sigma}+\widetilde{V}_{12 l 2} n_{2}\right)+\left(b_{2 \sigma}^{\dagger} b_{l \sigma}+b_{l \sigma}^{\dagger} b_{2 \sigma}\right)\left(\widetilde{V}_{1 l l l} n_{l-\sigma}+\widetilde{V}_{l 111} n_{2-\sigma}\right.\right. \\
& \left.\left.+\widetilde{V}_{12 l 2} n_{1}\right)+\left(b_{1 \sigma}^{\dagger} b_{2 \sigma}+b_{2 \sigma}^{\dagger} b_{1 \sigma}\right)\left[\tilde{V}_{1222}\left(n_{2-\sigma}+n_{1-\sigma}\right)+\widetilde{V}_{112 l} n_{l}\right]\right\}+\sum_{\sigma, \sigma^{\prime}}\left[\widetilde { V } _ { 1 2 2 l } \left(b_{l \sigma}^{\dagger} b_{2 \sigma^{\prime}}^{\dagger} b_{1 \sigma^{\prime}} b_{2 \sigma}+b_{1 \sigma}^{\dagger} b_{2 \sigma^{\prime}}^{\dagger} b_{l \sigma^{\prime}} b_{2 \sigma}\right.\right. \\
& \left.\left.+b_{l \sigma}^{\dagger} b_{1 \sigma^{\prime}}^{\dagger} b_{2 \sigma^{\prime}} b_{1 \sigma}+b_{2 \sigma^{\prime}}^{\dagger} b_{1 \sigma^{\prime}}^{\dagger} b_{l \sigma^{\prime}} b_{1 \sigma}\right)+\widetilde{V}_{1 l l 2}\left(b_{1 \sigma^{\prime}}^{\dagger} b_{l \sigma^{\prime}}^{\dagger} b_{2 \sigma^{\prime}} b_{l \sigma}+b_{2 \sigma^{\prime}}^{\dagger} b_{l \sigma^{\prime}}^{\dagger} b_{1 \sigma^{\prime}} b_{l \sigma}\right)\right]+\sum_{\sigma}\left[\tilde { V } _ { 1 2 2 l } \left(b_{2 \sigma}^{\dagger} b_{2-\sigma}^{\dagger} b_{l-\sigma} b_{1 \sigma}+b_{1 \sigma^{\prime}}^{\dagger} b_{l-\sigma^{\prime}}^{\dagger} b_{2-\sigma} b_{2 \sigma}\right.\right. \\
& \left.\left.+b_{1 \sigma}^{\dagger} b_{1-\sigma}^{\dagger} b_{l-\sigma} b_{2 \sigma}+b_{2 \sigma}^{\dagger} b_{l-\sigma}^{\dagger} b_{1-\sigma} b_{1 \sigma}\right)+\widetilde{V}_{1 l l 2}\left(b_{l \sigma}^{\dagger} b_{l-\sigma}^{\dagger} b_{2-\sigma} b_{1 \sigma}+b_{2 \sigma}^{\dagger} b_{2-\sigma}^{\dagger} b_{l-\sigma} b_{l \sigma}\right)\right] .
\end{aligned}
$$

In Eq. (B4), the $\tilde{V}_{i j k l}$ are the repulsion elements $\propto\left\langle i(1) j(2)\left|\frac{1}{r_{12}}\right| k(1) l(2)\right\rangle . \widetilde{K}_{i j}$ and $\widetilde{J}_{i j}$ stand for $\widetilde{V}_{i j i j}$ and $\widetilde{V}_{i j j i}$, respectively. The symmetry of the system (orbitals 1 and 2 are equivalent and interact identically with $l$ ) has been applied to reduce the number of different matrix elements in Eq. (B4). (Note that $\widetilde{V}_{11 l l}=\widetilde{V}_{1 l l 1} \equiv \widetilde{J}_{1 l}$ and the same for $\widetilde{V}_{1122}$ but this substitution is not carried through for clarity.) The expressions for the matrix elements in the Hamiltonian (B4) according to the transformation equations in Eq. (B3) are listed in Table I, along with approximate expressions for $\left|\frac{t}{\Delta}\right| \ll 1$. Note that $\widetilde{t}_{1 l}$ is exactly zero in the Anderson basis since it is an off-diagonal matrix element of the HF operator.

We want to relate the one-hole transfer element $\tilde{t}_{12}$ of Hamiltonian (B4) to the one-electron "kinetic transfer" integral $b_{12} \cdot{ }^{1}$ By its definition, $\widetilde{t}_{12}$ is the matrix element of the Hamiltonian (B4) between two one-hole configurations: one with the hole in the first Anderson magnetic orbital and the other with the hole in the second Anderson magnetic orbital, as depicted in the upper part of Fig. 9. The lower part of Fig. 9 represents exactly the same configurations in terms of electrons. The fact that a hole creation operator is actually defined as being exactly equal to an electron annihilation op- 


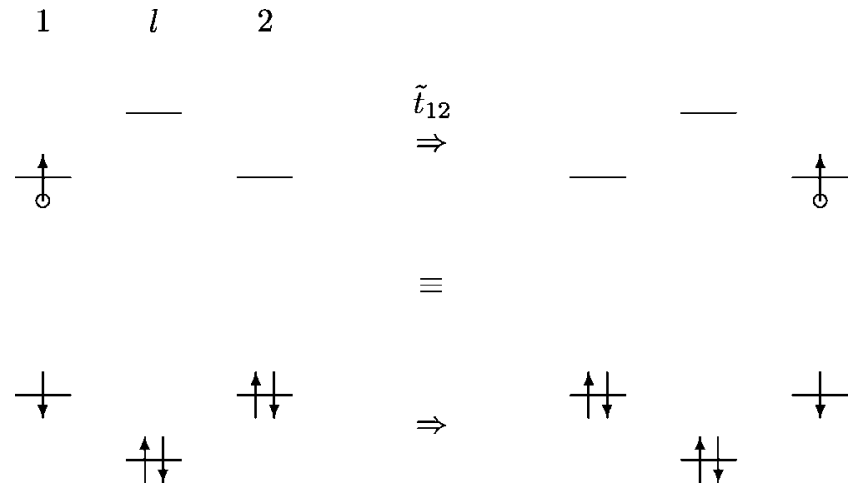

FIG. 9. Schematic representation of the one-hole transfer process (upper part) and its equivalent electron transfer process (lower part). The orbitals on the centra $1, l$, and 2 are $A_{1}, \Psi_{1+}$, and $A_{2}$ in Eqs. (21), (16), and (22), respectively.

erator ensures that the phase relationship between electron configurations is unambiguously defined. Thus, if the first hole configuration in Fig. 9 is written as $b_{1 \uparrow}\left|0_{h}\right\rangle$ and the second as $b_{2 \uparrow}\left|0_{h}\right\rangle$, then the expressions for the corresponding electron configurations are found by writing out $\left|0_{h}\right\rangle$ in terms of electron occupation. If we choose $\left|0_{h}\right\rangle$ $\equiv b_{1 \uparrow}^{\dagger} b_{1 \downarrow}^{\dagger} b_{2 \uparrow}^{\dagger} b_{2 \downarrow}^{\dagger} b_{l \uparrow}^{\dagger} b_{l \downarrow}^{\dagger}\left|0_{e}\right\rangle$, we have

$$
\begin{gathered}
b_{1 \uparrow}\left|0_{h}\right\rangle \equiv b_{1 \downarrow}^{\dagger} b_{2 \uparrow}^{\dagger} b_{2 \downarrow}^{\dagger} b_{l \uparrow}^{\dagger} b_{l \downarrow}^{\dagger}\left|0_{e}\right\rangle=-b_{2 \uparrow}^{\dagger} b_{1 \downarrow}^{\dagger} b_{2 \downarrow}^{\dagger} b_{l \uparrow}^{\dagger} b_{l \downarrow}^{\dagger}\left|0_{e}\right\rangle, \\
b_{2 \uparrow}\left|0_{h}\right\rangle \equiv b_{1 \uparrow}^{\dagger} b_{1 \downarrow}^{\dagger} b_{2 \downarrow}^{\dagger} b_{l \uparrow}^{\dagger} b_{l \downarrow}^{\dagger}\left|0_{e}\right\rangle .
\end{gathered}
$$

Taking the Hamiltonian matrix elements between both states at the same side of Eq. (B5), we find

$$
\tilde{t}_{12}=-\left(\tilde{F}_{12}^{c}+2 \tilde{V}_{1222}+2 \tilde{V}_{1 l 2 l}-\tilde{V}_{1 l l 2}\right)
$$

On the other hand, the Anderson parameter $b_{12}$ is the matrix element for the transfer of one electron between the metal sites in the field of the diamagnetic lattice, as depicted in Fig. 10. Therefore,

$$
b_{12}=\tilde{F}_{12}^{c}+2 \tilde{V}_{1 l 2 l}-\tilde{V}_{1 l l 2} .
$$

From Eqs. (B6) and (B7),

$$
b_{12}=-\left(\tilde{t}_{12}+2 \widetilde{V}_{1222}\right) \text {, }
$$

as used in Eq. (26).

\section{APPENDIX C: EVALUATION OF THE PERTURBATIONAL EXCHANGE ENERGY}

In calculating the exchange energy in the Anderson approach to a certain order of perturbation, one can follow two procedures. The first consists of separately calculating the perturbational energies of the lowest triplet and singlet states and subtracting one from the other. The second one makes use of an effective perturbation Hamiltonian that acts in the ground manifold, i.e., in the configurations with one hole in each magnetic orbital. This method is more convenient as it can easily be extended to systems with an unspecified number of magnetic centers. We have used the latter procedure to

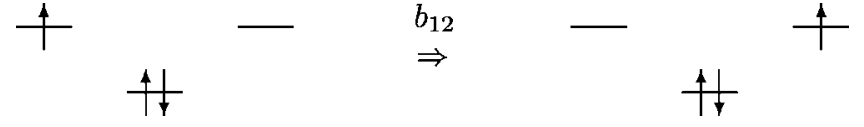

FIG. 10. Schematic representation of the electron transfer process with matrix element $b_{12}$. The orbitals on the centra $1, l$, and 2 are $A_{1}, \Psi_{1+}$, and $A_{2}$ in Eqs. (21), (16), and (22), respectively.

obtain exchange expressions up to fourth order, which are used in the plots. This appendix discusses some details of this procedure.

As was mentioned in the text, the Hamiltonian in Eq. (B4) is split into two parts: $H^{\text {holes }}=H^{0}+H^{\prime}$, where $H^{0}$ consists of the first line of Eq. (B4) and $H^{\prime}$ of the rest. The components of the effective perturbation Hamiltonian are then as specified in Eq. (23). The projection operators can be written out explicitly for the present model:

$$
P_{0}=\sum_{\sigma, \sigma^{\prime}} n_{1 \sigma} n_{2 \sigma^{\prime}}
$$

$$
\begin{aligned}
\frac{P}{a^{n}}= & \sum_{i=1}^{2}\left[\frac{n_{i \sigma} n_{i-\sigma}}{\left(\tilde{K}_{12}-\tilde{U}\right)^{n}}+\sum_{\sigma, \sigma^{\prime}} \frac{n_{i \sigma^{\prime}} n_{l \sigma^{\prime}}}{\left(\tilde{K}_{12}-\tilde{\Delta}-\tilde{K}_{1 l}\right)^{n}}\right] \\
& +\frac{n_{l \sigma} n_{l-\sigma}}{\left(\tilde{K}_{12}-2 \tilde{\Delta}-\tilde{U}^{\prime}\right)^{n}},
\end{aligned}
$$

with $n_{i \sigma}=b_{i \sigma}^{\dagger} b_{i \sigma}$. The expressions in Eq. (23) consist then of strings of creation and annihilation operators. Such strings of every length can, however, always be simplified to a maximum length of 4 using the anticommutation rules and the fact that in the ground manifold, one hole occupies each magnetic orbital. The resulting operators are connected with the on-site spin operators through the relation ${ }^{1,22}$

$$
\sum_{\sigma, \sigma^{\prime}} b_{1 \sigma^{\prime}}^{\dagger} b_{1 \sigma^{\prime}} b_{2 \sigma^{\prime}}^{\dagger} b_{2 \sigma}=2 \hat{\mathbf{S}}_{1} \hat{\mathbf{S}}_{2}+\frac{1}{2}
$$

In the final exchange Hamiltonian, constant terms are discarded as they do not contribute to the relative energy splitting. We can also see here that the effective perturbation Hamiltonians in Eq. (23) do not need to be made Hermitian explicitly because they all simplify to the form $\hat{\mathbf{S}}_{1} \hat{\mathbf{S}}_{2}$, which is Hermitian. The first- and second-order exchange Hamiltonians were given in the text in Eqs. (24) and (25). For the third order, we find 


$$
\begin{aligned}
& P_{0} H^{\prime} \frac{P}{a} H^{\prime} \frac{P}{a} H^{\prime} P_{0}=-4\left(\frac{\widetilde{V}_{1122}\left(\widetilde{t}_{12}+\widetilde{V}_{1222}\right)^{2}}{\left(\widetilde{U}-\widetilde{K}_{12}\right)^{2}}+\frac{2\left(\widetilde{t}_{1 l}+\widetilde{V}_{122 l}+\widetilde{V}_{12 l 2}\right) \widetilde{V}_{1 l l 2}\left(\widetilde{t}_{1 l}+\widetilde{V}_{1 l l l}\right)}{\left(\widetilde{\Delta}+\widetilde{K}_{1 l}-\widetilde{K}_{12}\right)\left(2 \widetilde{\Delta}+\widetilde{U}^{\prime}-\widetilde{K}_{12}\right)}+\frac{2 \widetilde{V}_{11 l l}\left(\widetilde{t}_{12}+\widetilde{V}_{1222}\right) \widetilde{V}_{1 l l 2}}{\left(\widetilde{U}-\widetilde{K}_{12}\right)\left(2 \widetilde{\Delta}+\widetilde{U}^{\prime}-\widetilde{K}_{12}\right)}\right. \\
& +\frac{2\left(\widetilde{t}_{12}+\widetilde{V}_{1222}\right)\left(\widetilde{t}_{1 l}+\widetilde{V}_{122 l}+\widetilde{V}_{12 l 2}\right)\left(\widetilde{t}_{1 l}+\widetilde{V}_{122 l}+\widetilde{V}_{l 111}\right)}{\left(\widetilde{U}-\widetilde{K}_{12}\right)\left(\widetilde{\Delta}+\widetilde{K}_{1 l}-\widetilde{K}_{12}\right)} \\
& \left.+\frac{\left[\tilde{V}_{122 l}^{2}+\left(\tilde{t}_{1 l}+\tilde{V}_{12 l 2}\right)^{2}\right]\left(\tilde{t}_{12}+\tilde{V}_{112 l}+\widetilde{J}_{1 l}\right)+2 \tilde{V}_{122 l}\left(\tilde{t}_{1 l}+\tilde{V}_{12 l 2}\right) \tilde{V}_{1 l l 2}}{\left(\widetilde{\Delta}+\widetilde{K}_{1 l}-\widetilde{K}_{12}\right)^{2}}\right) \hat{\mathbf{S}}_{1} \hat{\mathbf{S}}_{2}, \\
& P_{0} H^{\prime} P_{0} H^{\prime} \frac{P}{a^{2}} H^{\prime} P_{0}=-2 \widetilde{J}_{12}\left(\frac{2\left(\widetilde{t}_{12}+\widetilde{V}_{1222}\right)^{2}}{\left(\widetilde{U}-\widetilde{K}_{12}\right)^{2}}+\frac{2\left(\widetilde{V}_{122 l}^{2}+\left(\widetilde{t}_{1 l}+\widetilde{V}_{12 l 2}\right)^{2}\right)}{\left(\widetilde{\Delta}+\widetilde{K}_{1 l}-\widetilde{K}_{12}\right)^{2}}+\frac{\widetilde{V}_{1 l l 2}^{2}}{\left(2 \widetilde{\Delta}+\widetilde{U}^{\prime}-\widetilde{K}_{12}\right)^{2}}\right) \hat{\mathbf{S}}_{1} \hat{\mathbf{S}}_{2} \text {. }
\end{aligned}
$$

In a similar way, one can obtain the lengthy fourth-order exchange expressions.

${ }^{1}$ P. W. Anderson, Phys. Rev. 115, 2 (1959).

${ }^{2}$ W. Geertsma, Physica B 212, 50 (1995).

${ }^{3}$ W. Geertsma, J. Magn. Magn. Mater. 151, 67 (1995).

${ }^{4}$ W. Koshibae, Y. Ohta, and S. Maekawa, Phys. Rev. B 50, 3767 (1994)

${ }^{5}$ W. Geertsma and D. Khomskii, Phys. Rev. B 54, 3011 (1996).

${ }^{6}$ V. Yushankhai, M. Wolf, K.-H. Müller, R. Hayn, and H. Rosner, Phys. Rev. B 62, 14229 (2000).

${ }^{7}$ R. Hayn, V. A. Pashchenko, A. Stepanov, T. Masuda, and K. Uchinokura, Phys. Rev. B 66, 184414 (2002).

${ }^{8}$ M. V. Mostovoy and D. I. Khomskii, Phys. Rev. Lett. 89, 227203 (2002).

${ }^{9}$ E. Müller-Hartmann and A. Reischl, Eur. Phys. J. B 28, 173 (2002)

${ }^{10}$ R. M. Eremina, M. V. Eremin, V. N. Glazkov, H.-A. Krug von Nidda, and A. Loidl, Phys. Rev. B 68, 014417 (2003).

${ }^{11}$ M. V. Mostovoy and D. I. Khomskii, Phys. Rev. Lett. 92, 167201 (2004).

${ }^{12}$ P. M. Krstajić, F. M. Peeters, V. A. Ivanov, V. Fleurov, and K. Kikoin, Phys. Rev. B 70, 195215 (2004).

${ }^{13}$ A. M. Oleś, L. F. Feiner, P. Horsch, and G. Khaliullin, Phys. Status Solidi B 243, 89 (2006).

${ }^{14}$ R. Raghunathan, J.-P. Sutter, L. Ducasse, C. Desplanches, and S. Ramasesha, Phys. Rev. B 73, 104438 (2006).

${ }^{15}$ F. Tuczek and E. I. Solomon, Inorg. Chem. 32, 2850 (1993).

${ }^{16}$ C. A. Brown, G. J. Remar, R. L. Musselman, and E. I. Solomon, Inorg. Chem. 34, 688 (1995).

${ }^{17}$ H. Weihe, H. U. Güdel, and H. Toftlund, Inorg. Chem. 39, 1351 (2000).

${ }^{18}$ F. Tuczek and E. I. Solomon, Coord. Chem. Rev. 219-221, 1075 (2001).

${ }^{19}$ C. J. Calzado, J. Cabrero, J. P. Malrieu, and R. Caballol, J. Chem.
Phys. 116, 2728 (2002).

${ }^{20}$ C. J. Calzado, J. Cabrero, J. P. Malrieu, and R. Caballol, J. Chem. Phys. 116, 3985 (2002).

${ }^{21}$ P. W. Anderson, in Magnetism, edited by G. T. Rado and H. Suhl (Academic, New York, 1963), Vol. 1, p. 25.

${ }^{22}$ P. W. Anderson, Solid State Physics (Academic, London, 1963), Vol. 14, p. 99.

${ }^{23}$ W. Geertsma, Physica B 164, 241 (1990).

${ }^{24}$ J. Zaanen and G. A. Sawatzky, Can. J. Phys. 65, 1262 (1987).

${ }^{25}$ K.-I. Gondaira and Y. Tanabe, J. Phys. Soc. Jpn. 21, 1527 (1966).

${ }^{26}$ J. Zaanen, G. A. Sawatzky, and J. W. Allen, Phys. Rev. Lett. 55, 418 (1985).

${ }^{27}$ W. Geertsma, Ph.D. thesis, University of Groningen, 1979.

${ }^{28}$ L. F. Chibotaru, J.-J. Girerd, G. Blondin, T. Glaser, and K. Wieghardt, J. Am. Chem. Soc. 125, 12615 (2003).

${ }^{29}$ These matrices are found in Zaanen and Sawatzky (Ref. 24) and in Shen et al. (Ref. 30) but without the inclusion of $U^{\prime}$.

${ }^{30}$ Z.-X. Shen, J. W. Allen, J. J. Yeh, J.-S. Kang, W. Ellis, W. Spicer, I. Lindau, M. B. Maple, Y. D. Dalichaouch, M. S. Torikachvili, J. Z. Sun, and T. H. Geballe, Phys. Rev. B 36, 8414 (1987).

${ }^{31}$ P. J. Hay, J. C. Thibeault, and R. Hoffmann, J. Am. Chem. Soc. 97, 4884 (1975).

${ }^{32}$ See Eq. (B8), where $\tilde{t}_{12}$ (Table I) is always negative because $t \frac{c_{1}}{c_{2}}$ is always negative, as was mentioned in Sec. III A.

${ }^{33}$ This behavior really depends on the definition of the independent variable $\Delta=h_{11}+U-h_{l l}-U^{\prime}$ (see Sec. II). If $h_{11}-h_{l l}$, were held constant, then $\Delta$ grows with $U$ and the exchange interaction would reach zero when $U \rightarrow \infty$.

${ }^{34}$ J. Zaanen and G. A. Sawatzky, J. Solid State Chem. 88, 8 (1990).

${ }^{35}$ In fact, for small $\Delta$ a lowering of $U^{\prime}$ makes the VBCI approximation even worse, as can be seen in the third column of Fig. 5. 